\title{
MONODROMY FILTRATIONS AND THE TOPOLOGY OF TROPICAL VARIETIES
}

\author{
DAVID HELM AND ERIC KATZ
}

\begin{abstract}
We study the topology of tropical varieties that arise from a certain natural class of varieties. We use the theory of tropical degenerations to construct a natural, "multiplicity-free" parameterization of $\operatorname{Trop}(X)$ by a topological space $\Gamma_{X}$ and give a geometric interpretation of the cohomology of $\Gamma_{X}$ in terms of the action of a monodromy operator on the cohomology of $X$. This gives bounds on the Betti numbers of $\Gamma_{X}$ in terms of the Betti numbers of $X$ which constrains the topology of $\operatorname{Trop}(X)$. We also obtain a description of the top power of the monodromy operator acting on middle cohomology of $X$ in terms of the volume pairing on $\Gamma_{X}$.
\end{abstract}

\section{INTRODUCTION}

Let $\mathcal{O}$ be a discrete valuation ring with field of fractions $K$. Tropicalization is a procedure which takes as input a $d$-dimensional subvariety of an algebraic torus over $K, X \subset\left(K^{*}\right)^{n}$, and associates to it a balanced weighted rational $d$-dimensional polyhedral complex, $\operatorname{Trop}(X) \subseteq \mathbb{R}^{n}$. Several questions naturally arise in this framework; for instance, one may ask what combinatorial properties of $\operatorname{Trop}(X)$ correspond to geometric properties of $X$. One may also ask what constraints being a tropicalization places on the topology of a polyhedral complex. In $[\mathrm{H}]$, Hacking proved that if $X$ is a subvariety of $\left(\mathbb{C}^{*}\right)^{n}$ satisfying a certain genericity condition, then the link of the fan $\operatorname{Trop}(X)$ only has reduced rational homology in the top dimension. Hacking's result holds for a number of examples, including generic intersections of ample hypersurfaces in projective toric varieties. In [Sp2, Sec. 10], Speyer showed that if $C$ is a genus $g$ curve in $\left(K^{*}\right)^{n}$ satisfying a genericity condition then there exists a balanced metric graph $\Gamma$ with $b_{1}(\Gamma) \leq g$ and a parameterization $i: \Gamma \rightarrow \operatorname{Trop}(C)$ that is affine-linear on edges. Our results include an analogue of Hacking's result for varieties defined over $K$ or as a higher-dimensional generalization of Speyer's result. One may consider the monodromy action of $\operatorname{Gal}\left(K^{\mathrm{sep}} / K\right)$ on the étale cohomology $H_{\text {et }}^{*}\left(X_{K^{\text {sep }}}, \mathbb{Q}_{l}\right)$ and ask what properties of the monodromy action are encoded in $\operatorname{Trop}(X)$. This is the algebraic analogue of the monodromy action of a family of varieties defined over a punctured disk. The work of the second-named author with Hannah Markwig and Thomas Markwig [KMM] relating the valuation of the $j$-invariant of a plane elliptic curve to the length of the cycle in its tropicalization can be seen in this light. We give a generalization of that result.

All of our results require that the variety $X$ be schön, a natural condition introduced in $[\mathrm{T}$ and generalized by $\mathrm{LQ}$ to the nonconstant coefficient case. This condition means that the ambient torus $\left(K^{*}\right)^{n}$ may be compactified to a toric scheme $\mathbb{P}$ over an extension of $\operatorname{Spec} \mathcal{O}$ such that the intersection of $\mathcal{X}=\bar{X} \subset \mathbb{P}$ 
with each open torus orbit $U_{P}$ is smooth of the expected dimension. For appropriate $\mathbb{P}, \mathcal{X}$ is then a simple normal crossings degenerations of $X$ (c.f. 3.10). The construction of the desired toric scheme $\mathbb{P}$ follows from Proposition 2.3 a technical result in polyhedral geometry which has not appeared, to our knowledge, in the literature before.

The existence of such simple normal crossings degenerations for a schön $X$ allows us to construct a natural "parameterizing space" $\Gamma_{X}$. This generalizes a construction introduced by Speyer $\left[\mathrm{Sp} 2\right.$ when $X$ has dimension 1 . The space $\Gamma_{X}$ is closely related to the dual complex of an appropriate degeneration $\mathcal{X}$ of $X$; in this guise it already appears implicitly in [H], as well as in $[\mathrm{HKT}$. Kontsevich-Soibelman use rigid analytic techniques to construct a similar polyhedral complex, with an integral affine structure, from a suitable degeneration of $X$ in [KS06.

The space $\Gamma_{X}$ we construct is independent of a choice of model $\mathcal{X}$ for $X$; it depends only on $X$ and its embedding in the torus. Moreover, $\Gamma_{X}$ comes equipped with a canonical map to $\operatorname{Trop}(X)$. A choice of sufficiently fine triangulation of $\operatorname{Trop}(X)$ gives $\Gamma_{X}$ the structure of a polyhedral complex. When $\Gamma_{X}$ is viewed in such a way, the natural parameterization $\Gamma_{X} \rightarrow \operatorname{Trop}(X)$ is affine-linear on polyhedra. This parameterization has several nice properties. For instance, it is natural under monomial morphisms: if $X$ and $Y$ are schön subvarieties of tori $T$ and $T^{\prime}$ and $\phi: T \rightarrow T^{\prime}$ is a homomorphism taking $X$ to $Y$, then there is an induced map of complexes $\Gamma_{X} \rightarrow \Gamma_{Y}$ that commutes with parameterizations. Moreover, $\Gamma_{X}$ satisfies a balancing condition analogous to the one satisfied by all tropical varieties. Finally, it is "not far" from $\operatorname{Trop}(X)$ : if the intersections of $\mathcal{X}$ with open torus orbits $U_{P}$ in $\mathbb{P}$ satisfy certain connectedness hypotheses, we may equate the cohomology of $\Gamma_{X}$ and $\operatorname{Trop}(X)$ in certain degrees. We hope that parameterizing complexes will be seen as a fundamental object in tropical geometry.

Our main results (principally Theorem 6.1, Corollary 6.3, and Proposition 7.4) relate the cohomology of $\Gamma_{X}$ to geometric invariants of $X$. In particular we consider the étale cohomology $H_{\text {et }}^{*}\left(X_{K^{\text {sep }}}, \mathbb{Q}_{l}\right)$; this cohomology comes equipped with a natural filtration, called the weight filtration. We construct a natural isomorphism between the "weight 0 " subquotient $W_{0} H_{\text {ét }}^{r}\left(X_{K^{\text {sep }}}, \mathbb{Q}_{l}\right)$ arising from this filtration and the cohomology $H^{r}\left(\Gamma_{X}, \mathbb{Q}_{l}\right)$ of $\Gamma_{X}$. We then use this to show that if $X$ is the generic intersection of ample hypersurfaces in a toric scheme $\mathbb{P}$, then $H^{r}\left(\operatorname{Trop}(X), \mathbb{Q}_{l}\right)$ vanishes for $1 \leq r<\operatorname{dim} X$, a non-constant coefficient analogue of Hacking's result. We also prove results about the monodromy action on the middle cohomology $H_{\text {ét }}^{n}\left(X_{K^{\text {sep }}}, \mathbb{Q}_{l}\right)$.

The main tool we use is the Rapoport-Zink weight spectral sequence [RZ]. Under the schön condition, after a finite base-extension $\mathcal{O}^{\prime}$ of $\mathcal{O}$, we may compactify the ambient torus to a toric scheme $\mathbb{P}$ defined over $\mathcal{O}^{\prime}$ so that the central fiber of the closure $\mathcal{X}$ of $X$ in $\mathbb{P}$ is a divisor with simple normal crossings. The divisor, a degeneration of $X$, has a stratification coming from intersections of its irreducible components. The Rapoport-Zink spectral sequence then gives a very explicit formula for the cohomology on $X$, together with its weight filtration, in terms of these strata. The $E_{1}$-term of the weight spectral sequence is formed from the cohomology groups of the strata with boundary maps built from the data of restriction maps and Gysin maps. The spectral sequence converges to the cohomology of the general fiber, and the induced filtration is the weight filtration. Moreover, the weight spectral sequence degenerates at $E_{2}$. We thus obtain an explicit description of the 
smallest nontrivial piece of the filtration which is isomorphic to the cohomology groups of $\Gamma_{X}$.

It is interesting to compare this result to results of Berkovich $[\mathrm{B}$. on rigid analytic spaces. In particular, Berkovich shows that the cohomology group $H_{\text {ét }}^{r}\left(\bar{X}_{K^{\text {sep }}}, \mathbb{Q}_{l}\right)_{-r}$ arising in our result is isomorphic to the cohomology of the Berkovich space $X^{\text {an }}$ attached to $X$. Our result thus suggests a strong link between $\Gamma_{X}$ and $X^{\text {an }}$. In fact, Speyer Sp1 constructs a natural map from $X^{\text {an }}$ to $\operatorname{Trop}(X)$. This map factors through the map $\Gamma_{X} \rightarrow \operatorname{Trop}(X)$, and it is natural to ask if the resulting map $X^{\text {an }} \rightarrow \Gamma_{X}$ map is a homotopy equivalence. Links between tropical geometry and rigid geometry have also appeared in works of Einsiedler-Kapranov-Lind [EKL, and Payne $[\mathrm{P}$.

Under additional hypotheses, one can relate the results above to questions involving monodromy. A variety defined over Spec $K$ is analogous to a family of varieties defined over a punctured disk. The fundamental group of the punctured disk acts on the cohomology of a general fiber of such a family by monodromy. The analogue of this monodromy action for varieties over Spec $K$ is the action of the

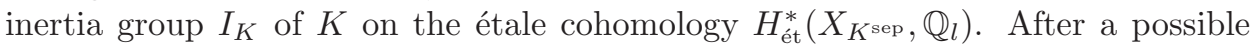
finite base-extension of $\mathcal{O}$, this action is unipotent, and is given by the monodromy operator

$$
N: H_{\text {ét }}^{i}\left(X_{K^{\text {sep }}}, \mathbb{Q}_{l}\right) \rightarrow H_{\text {ét }}^{i}\left(X_{K^{\text {sep }}}, \mathbb{Q}_{l}(-1)\right)
$$

an endomorphism of the étale cohomology that is essentially the (matrix) logarithm of the action. (We refer the reader to section 7 for precise definitions). The action of $N$ induces an increasing filtration on the cohomology. The weight-monodromy conjecture asserts that this filtration coincides (up to a shift in degree) with the weight filtration described above. Although it is not completely settled, this conjecture is known to be true in many cases of interest; for instance, it is known $X$ is a curve, surface, or an abelian variety. Ito [I] has proven the weight-mondromy conjecture when $\mathcal{O}$ has equal characteristic. Thus, in these situations, one can interpret Theorem 6.1 as an isomorphism between the cohomology of $\Gamma_{X}$ and the smallest nontrivial piece of the monodromy filtration of the cohomology of $\bar{X}_{K}$, the closure of $X_{K}$ in the generic fiber of $\mathbb{P}$ :

$$
H^{r}\left(\Gamma_{X}, \mathbb{Q}_{l}\right) \cong H_{\text {ét }}^{r}\left(\bar{X}_{K^{\text {sep }}}, \mathbb{Q}_{l}\right)_{-r} .
$$

As a consequence, Corollary 7.3 bounds the Betti numbers of $\Gamma_{X}$ in terms of those of $X$ :

$$
b_{r}\left(\Gamma_{X}\right) \leq \frac{1}{r+1} b_{r}(X)
$$

generalization Speyer's result on curves.

We apply our techniques to get a description of some of the monodromy action and not just of the monodromy filtration. In Proposition 7.4 we give an interpretation of the top power of monodromy operator acting on the middle-dimensional cohomology

$$
N^{d}: H_{\text {ét }}^{d}\left(X_{K^{\text {sep }}}, \mathbb{Q}_{l}\right)_{d} / H_{\text {ét }}^{d}\left(X_{K^{\text {sep }}}, \mathbb{Q}_{l}\right)_{d-1} \rightarrow H_{\text {ét }}^{d}\left(X_{K^{\text {sep }}}, \mathbb{Q}_{l}\right)_{-d}(-d)
$$

using the isomorphisms

$$
\begin{aligned}
H_{\text {ét }}^{d}\left(X_{K^{\mathrm{sep}}}, \mathbb{Q}_{l}\right)_{d} / H_{\text {êt }}^{d}\left(X_{\left.K_{\mathrm{sep}}, \mathbb{Q}_{l}\right)_{d-1}}\right. & \cong H_{d}\left(\Gamma, \mathbb{Q}_{l}\right)(-d) \\
H_{\text {êt }}^{d}\left(\bar{X}_{K^{\mathrm{sep}},}, \mathbb{Q}_{l}\right)_{-d} & \cong H^{d}\left(\Gamma_{X}, \mathbb{Q}_{l}\right) .
\end{aligned}
$$


The operator can be viewed as a bilinear pairing on $H_{d}\left(\Gamma_{X}, \mathbb{Q}_{l}\right)$ in which case it is the volume pairing that takes a pair of top-dimensional cycles to the oriented volume of their intersection. This specializes to the length pairing in the case of curves. In the case of genus 1 curves, by a straightforward application of the conductor-discriminant formula, we are able to recover the spatial schön analogue of a result proved by the second-named author with H. Markwig andT. Markwig KMM: the valuation of the $j$-invariant of an elliptic curve $X$ with potentially multiplicative reduction is equal to $-a$ where $a$ is the length of the unique cycle in $\Gamma_{X}$.

Our techniques are very similar to those of Hacking and Speyer. Hacking uses a spectral sequence coming from a weight filtration on a complex of differential forms while we use the Rapoport-Zink spectral sequence. Speyer's results use a resolution of the structure sheaf of a degeneration of a curve $C$ coming from a stratification induced by a toric scheme while we use a locally constant sheaf.

We should mention some related results. In GS, Gross and Siebert construct a scheme $X_{0}$ from an integer affine manifold and a toric polyhedral decomposition. If $X_{0}$ is embedded in a family $\mathcal{X}$ over $\mathbb{C}[[t]]$, they are able to determine the limit mixed Hodge structure in terms of the combinatorial data. In KS10, the second-named author and Stapledon give a description of the Hodge-Deligne polynomial of the limit mixed Hodge structure of a schön family of varieties over a punctured disk in terms of $\operatorname{Trop}(X)$ and initial degenerations of $X$.

We would like to thank Brian Conrad, Richard Hain, Kalle Karu, Sean Keel, Sam Payne, Zhenhua Qu, Bernd Siebert, Martin Sombra, David Speyer, Alan Stapledon, and Bernd Sturmfels for valuable discussions.

\section{TORIC SCHEMES}

We begin by reviewing a construction that attaches a degenerating family of toric varieties over a discrete valuation ring to a rational polyhedral complex in $\mathbb{R}^{n}$. This has appeared several times in the literature Sp1 [NS $[\mathrm{S}$. We follow the approach of [NS] here. Fix a discrete valuation ring $\mathcal{O}$, with field of fractions $K$ and residue field $k$, and a uniformizer $\pi$ of $\mathcal{O}$.

Definition 2.1. A rational polyhedral complex in $\mathbb{R}^{n}$ is a collection $\Sigma$ of finitely many convex rational polyhedra $P \subset \mathbb{R}^{n}$ with the following properties:

- If $P \in \Sigma$ and $P^{\prime}$ is a face of $P$, then $P^{\prime}$ is in $\Sigma$.

- If $P, P^{\prime} \in \Sigma$ then $P \cap P^{\prime}$ is a face of both $P$ and $P^{\prime}$.

If, in addition, the union $\bigcup_{P \in \Xi} P$ is equal to $\mathbb{R}^{n}$, then $\Sigma$ is said to be complete.

Given a $\Sigma$ as above, we can construct a fan $\tilde{\Sigma}$ in $\mathbb{R}^{n} \times \mathbb{R}_{\geq 0}$ as follows: for each $P \in \Sigma$ let $\tilde{P}$ be the closure in $\mathbb{R}^{n} \times \mathbb{R}_{\geq 0}$ of the set

$$
\left\{(x, a) \subset \mathbb{R}^{n} \times \mathbb{R}_{>0}: \frac{x}{a} \in P\right\} .
$$

Then $\tilde{P}$ is a rational polyhedral cone in $\mathbb{R}^{n} \times \mathbb{R}_{>0}$. Its facets come in two types:

- cones of the form $\tilde{P}^{\prime}$, where $P^{\prime}$ is a facet of $P$, and

- the cone $P_{0}=\tilde{P} \cap\left(\mathbb{R}^{n} \times\{0\}\right)$, which is the limit as a goes to zero of the polyhedron $a P$ in $\mathbb{R}^{n}$.

We let $\tilde{\Sigma}$ be the collection of cones of the form $\tilde{P}$ and $P_{0}$ for $P$ in $\Sigma$. If $\Sigma$ is a subcomplex of a complete rational polyhedral complex, we say that $\Sigma$ is completable 
and, by Corollary 3.12 of [BGS, $\tilde{\Sigma}$ is a rational polyhedral fan in $\mathbb{R}^{n} \times \mathbb{R}_{\geq 0}$. Note that $\Sigma=\tilde{\Sigma} \cap\left(\mathbb{R}^{n} \times\{1\}\right)$. On the other hand the fan $\Sigma_{0}$ given by $\tilde{\Sigma} \cap\left(\mathbb{R}^{n} \times\{0\}\right)$ is the limit as $a$ approaches zero of the polyhedral complexes $a \Sigma$.

Remark 2.2. In fact, by results of [BGS], the association $\Sigma \mapsto \tilde{\Sigma}$ defines a bijection between the set of complete polyhedral complexes in $\mathbb{R}^{n}$ and the set of complete fans in $\mathbb{R}^{n} \times \mathbb{R}_{\geq 0}$ for which every cone contained in $\mathbb{R}^{n} \times\{0\}$ is the boundary of a cone that meets $\mathbb{R}^{N} \times \mathbb{R}_{>0}$. Its inverse is $\tilde{\Sigma} \mapsto \tilde{\Sigma} \cap\left(\mathbb{R}^{n} \times\{1\}\right)$.

Let $X(\tilde{\Sigma})_{\mathbb{Z}}$ be the toric scheme over $\mathbb{Z}$ associated to the fan $\tilde{\Sigma}$. (The construction associating a toric variety to a fan is usually given over a field, but works just as well with coefficients in $\mathbb{Z}$.) Projection from $\mathbb{R}^{n} \times \mathbb{R}_{\geq 0}$ to $\mathbb{R}_{\geq 0}$ induces a map of fans from $\tilde{\Sigma}$ to the fan $\left\{0, \mathbb{R}_{\geq 0}\right\}$ associated to $\mathbb{A}_{\mathbb{Z}}^{1}$. This gives rise to a map of toric varieties $\pi_{\mathbb{Z}}: X(\tilde{\Sigma})_{\mathbb{Z}} \rightarrow \mathbb{A}_{\mathbb{Z}}^{1}$. As remarked in [NS, this map is flat and torus equivariant. Let $\iota: \operatorname{Spec} \mathcal{O} \rightarrow \mathbb{A}_{\mathbb{Z}}^{1}$ be the map corresponding to the map $\mathbb{Z}[t] \rightarrow \mathcal{O}$ that takes $t$ to $\pi$. We let $X(\tilde{\Sigma})$ be the scheme over $\mathcal{O}$ obtained by base change from $X(\tilde{\Sigma})_{\mathbb{Z}}$ via $\iota$, and let $\pi: X(\tilde{\Sigma}) \rightarrow \mathcal{O}$ be the projection.

We summarize results of [NS] concerning this construction:

- The general fiber $X(\tilde{\Sigma}) \times_{\operatorname{Spec} \mathcal{O}} \operatorname{Spec} K$ is isomorphic to the toric variety over $K$ associated to $\Sigma_{0}$.

- If $\Sigma$ is integral, i.e. the vertices of every polyhedron in $\Sigma$ lie in $\mathbb{Z}^{n}$, then the special fiber $X(\tilde{\Sigma})_{k}=X(\tilde{\Sigma}) \times_{\operatorname{Spec} \mathcal{O}} \operatorname{Spec} k$ is reduced.

- There is an inclusion-reversing bijection between closed torus orbits in $X(\tilde{\Sigma})_{k}$ and polyhedra $P$ in $\Sigma$; the irreducible components of $X(\tilde{\Sigma})_{k}$ correspond to vertices in $\Sigma$; the intersection of a collection of irreducible components corresponds to the smallest polyhedron in $\Sigma$ containing all of their vertices.

Note that adjoining a $d$ th root of $\pi$ to $\mathcal{O}$ has the effect of rescaling $\Sigma$ by $d$; that is, if $\mathcal{O}^{\prime}=\mathcal{O}\left[\pi^{\frac{1}{d}}\right]$, then the base change of the family $X(\tilde{\Sigma}) \rightarrow \mathcal{O}$ is the family $X(\widetilde{(d \Sigma)}) \rightarrow \mathcal{O}^{\prime}$. In particular, given any toric scheme coming from a polyhedral complex $\Sigma$, we can choose $d$ such that $d \Sigma$ is integral; after taking a suitable ramified base change of $\mathcal{O}$ the special fiber of the family $X(\tilde{\Sigma}) \rightarrow \mathcal{O}$ will be reduced.

We will be particularly interested in degenerations of toric varieties in which the special fiber is a divisor with simple normal crossings. These are easy to construct, because the boundary of a smooth toric variety is always a divisor with simple normal crossings. We make use of the following result in polyhedral combinatorics:

Proposition 2.3. Let $\Sigma$ be a complete rational polyhedral complex in $\mathbb{R}^{n}$. There exists an integer $d$, and a subdivision $\Sigma^{\prime}$ of $d \Sigma$ such that the general fiber of the scheme $X\left(\tilde{\Sigma}^{\prime}\right)$ is a smooth toric variety and the special fiber of $X\left(\tilde{\Sigma}^{\prime}\right)$ is a divisor with simple normal crossings. Moreover, if the recession fan $\Sigma_{0}$ is already simplicial and unimodular, $\Sigma^{\prime}$ can be chosen to have $\Sigma_{0}^{\prime}=\Sigma_{0}$.

Proof. Choose an integer $l_{1}$ such that $l_{1} \Sigma$ is integral. Fulton [F, Sec. 2.6] gives an algorithm for constructing a subdivision $\tilde{\Sigma}^{\prime}$ of the fan $\widetilde{l_{1} \Sigma}$ such that all the cones of $\tilde{\Sigma}_{1}$ are simplicial and unimodular. Pick an integer $l_{2}$ sufficiently divisible so $\Sigma_{1}=\tilde{\Sigma}^{\prime} \cap\left(\mathbb{R}^{n} \times\left\{l_{2}\right\}\right)$ is integral. $\Sigma_{1}$ is a subdivision of $\mathbb{R}^{n}$ with the property that each of its recession cones is simplicial. Because $\tilde{\Sigma}_{1}$ is simplicial each of its cones is of the form $\tilde{P}+Q_{0}$ where $P$ is a simplex in $\Sigma_{1}, Q_{0}$ is a cone in the recession 
fan $\left(\Sigma_{1}\right)_{0}$, and $\tilde{P} \cap Q_{0}=\{0\}$. Consequently, the corresponding polyhedron of $\Sigma_{1}$ is $\left(\tilde{P}+Q_{0}\right) \cap\left(\mathbb{R}^{n} \times\left\{l_{2}\right\}\right)=P+Q_{0}$. For a polyhedron $F$ in $\mathbb{R}^{k}$, let $N_{F}=$ $\mathbb{Z}^{k} \cap \operatorname{Span}_{\mathbb{R}}(F-w)$ where $w$ is a point of $F$. If $F$ is a rational polytope, then $N_{F}$ has the property a basis of it can be extended to a basis of $\mathbb{Z}^{k}$.

We claim $N_{P}+N_{Q_{0}}=N_{P+Q_{0}}$ for every cone $\tilde{P}+Q_{0}$ of $\tilde{\Sigma}_{1}$. It is clear that $N_{P}+N_{Q_{0}} \subseteq N_{P+Q_{0}}$. The inclusion $\mathbb{Z}^{n} \hookrightarrow \mathbb{Z}^{n+1}=\mathbb{Z}^{n} \times \mathbb{Z}$ given by $x \mapsto\left(x, l_{2}\right)$ identifies $N_{P+Q_{0}}$ with the intersection of $N_{\tilde{P}+Q_{0}}$ with $\mathbb{Z}^{n} \times\left\{l_{2}\right\}$. Since $\tilde{P}+Q_{0}$ is unimodular, $N_{\tilde{P}}+N_{Q_{0}}$ is equal to $N_{\tilde{P}+Q_{0}}$. Therefore, if $x \in N_{P+Q_{0}},\left(x, l_{2}\right)=$ $x_{\tilde{P}}+x_{Q_{0}}$ where $x_{\tilde{P}} \in \tilde{P}$ and $x_{Q_{0}} \in Q_{0}$. Since the last coordinate of $x_{Q_{0}}$ is 0 , $x_{\tilde{P}}=\left(x_{P}, l_{2}\right)$ for $x_{P} \in N_{P}$. It follows that $x=x_{P}+x_{Q_{0}}$.

Let $\Sigma_{1}^{b}$ be the union of the bounded polyhedra of $\Sigma_{1}$. By an important step of the proof of semi-stable reduction [KKMS, Ch. 3, Thm 4.1], there is an integer $l_{2}$ and a unimodular triangulation $\Sigma_{1}^{b^{\prime}}$ of $l_{2} \Sigma_{1}^{b}$. This induces a subdivision of $l_{2} \Sigma_{1}$ where the polyhedra whose relative interior is contained in the relative interior of $l_{2}\left(P+Q_{0}\right)$ are of the form $P^{\prime}+Q_{0}$ where $P^{\prime}$ is a simplex in $\Sigma_{1}^{b^{\prime}}$ whose relative interior is contained in the the relative interior of $l_{2} P$. We call this subdivision $\Sigma^{\prime}$. It is simplicial by construction. We claim that it is also unimodular. It suffices to show that maximal cones in $\tilde{\Sigma}^{\prime}$ are unimodular. Let $\tilde{P}^{\prime}+Q_{0}$ be a maximal cone in $\tilde{\Sigma}^{\prime}$. Then the relative interior of $P^{\prime}$ is contained in the relative interior of $l_{2} P$ with $\operatorname{dim} P=\operatorname{dim} P^{\prime}$. Since $P^{\prime}$ is unimodular, its $\mathbb{Z}$-affine span is $N_{P^{\prime}}$. Consequently, since $N_{P^{\prime}}+N_{Q_{0}}=N_{P}+N_{Q_{0}}=N_{P+Q_{0}}$, we see that any element of $N_{P+Q_{0}}$ can be written as integer combination $\sum m_{i} v_{i}+\sum n_{i} w_{i}$ where $v_{i}$ are vertices of $P^{\prime}, w_{i}$ are the primitive vectors along the rays of $Q_{0}$, and $\sum m_{i}=1$. Consequently, any element of $N_{P+Q_{0}} \times\{1\}$ can be written as an integer combination

of the primitive vectors along the rays of $\tilde{P}^{\prime}+Q_{0}$. Consequently these vectors generate $N_{\tilde{P}+Q_{0}} \subset \mathbb{Z}^{n+1}$. Therefore $\tilde{P}^{\prime}+Q_{0}$ is smooth.

If $\Sigma_{0}$ was simplicial and unimodular to begin with, none of these steps would have affected the cones $Q_{0}$ of $\Sigma_{0}$

The upshot is that $X\left(\tilde{\Sigma}^{\prime}\right)$ is a smooth toric variety with a birational morphism $X\left(\tilde{\Sigma}^{\prime}\right) \rightarrow X(\tilde{\Sigma})$. The induced map $X\left(\tilde{\Sigma}^{\prime}\right) \rightarrow \mathcal{O}$ is the toric scheme associated to the integral polyhedral complex $\Sigma^{\prime}$. The general fiber of $X\left(\tilde{\Sigma}^{\prime}\right)$ over $\mathcal{O}$ corresponds to the fan $\Sigma_{0}^{\prime}$, and is therefore smooth. The special fiber is a union of irreducible components of the boundary of $X\left(\tilde{\Sigma}^{\prime}\right)$ and is therefore a divisor of $X\left(\tilde{\Sigma}^{\prime}\right)$ with simple normal crossings.

\section{Tropical Degenerations}

We now describe the applications of tropical geometry to the study of degenerations of varieties over $K$. These techniques have their origins in the Speyer's thesis Sp1. The approach we take here is due to Tevelev [T] in the "constant coefficient case"; the extension of Tevelev's work to the case of an arbitrary DVR done by Luxton and $\mathrm{Qu} \mathrm{LQ}$.

Let $\bar{K}$ be an algebraic closure of $K$. There is a unique valuation

$$
\text { ord }: \bar{K} \rightarrow \mathbb{Q}
$$

such that $\operatorname{ord}(\pi)=1$. 
Let $\mathcal{T} \cong \mathbb{G}_{m}^{n}$ be a split $n$-dimensional torus over $\mathcal{O}$, and let $T=\mathcal{T} \times_{\mathcal{O}} K$ be the corresponding torus over $K$. The valuation ord gives rise to a map

$$
\text { val }: \mathcal{T}(\bar{K}) \rightarrow \mathbb{Q}^{n},
$$

by fixing an isomorphism of $\mathcal{T}$ with $\mathbb{G}_{m}^{n}$ (and hence an isomorphism of $\mathcal{T}(\bar{K})$ with $\left(\bar{K}^{*}\right)^{n}$.) Let $X$ be a closed subvariety of $T$, defined over $K$.

Definition 3.1 (EKL, 1.2.1). : The tropical variety $\operatorname{Trop}(X)$ associated to $X$ is the closure of $\operatorname{val}(X(\bar{K}))$ in $\mathbb{R}^{n}$.

Given such an $X$, one can ask for a well-behaved compactification $\bar{X}$ of $X$, and a well-behaved degeneration of $\bar{X}$ over $\mathcal{O}$. The problem of finding such a degeneration is intimately connected to the set $\operatorname{Trop}(X)$.

Let $\Sigma$ be a completable rational polyhedral complex in $\mathbb{R}^{n}$, and let $\mathbb{P}$ be the corresponding toric scheme over $\mathcal{O}$. Identify the group of cocharacters of $\mathcal{T}$ with $\mathbb{Z}^{n}$ in $\mathbb{R}^{n}$; this identifies $\mathbb{T}$ with the open torus orbit on $\mathbb{P}$.

We can thus take the closure of $\mathcal{X}$ of $X$ in $\mathbb{P}$. By [Sp1, 2.4.1, the scheme $\mathcal{X}$ is proper over $\mathcal{O}$ if, and only if, $\operatorname{Supp} \Sigma$ contains $\operatorname{Trop}(X)$. We assume henceforth that $\operatorname{Supp} \Sigma$ contains $\operatorname{Trop}(X)$. Let $\bar{X}$ be the fiber of $\mathcal{X}$ over $K$, and $\mathcal{X}_{k}$ be the special fiber of $\mathcal{X}$. The natural multiplication map

$$
\mathcal{T} \times_{\mathcal{O}} \mathbb{P} \rightarrow \mathbb{P}
$$

restricts to a multiplication map

$$
m: \mathcal{T} \times_{\mathcal{O}} \mathcal{X} \rightarrow \mathbb{P} .
$$

Definition 3.2. The pair $(X, \mathbb{P})$ is tropical if the map

$$
m: \mathcal{T} \times_{\mathcal{O}} \mathcal{X} \rightarrow \mathbb{P}
$$

is faithfully flat, and $\mathcal{X} \rightarrow \mathcal{O}$ is proper.

We then have the following, due to Tevelev [T] in the constant coefficient case. In the non-constant coefficient case they can be found in LQ.

Proposition 3.3. A subvariety $X \subset\left(\bar{K}^{*}\right)^{n}$ admits a tropical pair $(X, \mathbb{P})$.

Proposition 3.4. Suppose $(X, \mathbb{P})$ is tropical and let $\mathbb{P}^{\prime} \rightarrow \mathbb{P}$ be a morphism of toric schemes corresponding to a refinement $\Sigma^{\prime}$ of $\Sigma$. Then $\left(X, \mathbb{P}^{\prime}\right)$ is also tropical.

Proposition 3.5. If $(X, \mathbb{P})$ is a tropical pair then $\operatorname{Supp} \Sigma=\operatorname{Trop}(X)$.

Following Speyer $([\mathrm{Sp} 1,2.4)$ If $(X, \mathbb{P})$ is a tropical pair, we call $\bar{X}$ a tropical compactification of $X$, and $\mathcal{X}_{k}$ a tropical degeneration of $X$.

The combinatorics of the special fiber of a tropical degeneration of $X$ is closely related to the combinatorics of $\operatorname{Trop}(X)$. In particular if $(X, \mathbb{P})$ is a tropical pair, and $\mathcal{X}$ is the corresponding tropical degeneration, then a polyhedron $P$ of $\Sigma$ corresponds to the closure of a torus orbit in the special fiber of $\mathbb{P}$. Call this torus orbit closure $\mathbb{P}_{P}$. Then the intersection $\mathcal{X}_{P}$ of $\mathcal{X}$ with $\mathbb{P}_{P}$ is a closed subscheme of $\mathcal{X}_{k}$. Moreover, if $P$ and $P^{\prime}$ are polyhedra of $\Sigma$, and $Q$ is the smallest polyhedron in $\Sigma$ containing both $P$ and $P^{\prime}$, then the intersection of $\mathcal{X}_{P}$ and $\mathcal{X}_{P^{\prime}}$ is $\mathcal{X}_{Q}$.

Let $U_{P}$ be the open torus orbit corresponding to $P$. Fix a point $p$ in $U_{P}$, and consider the fiber over $p$ of the multiplication map

$$
m: \mathcal{T} \times_{\mathcal{O}} \mathcal{X} \rightarrow \mathbb{P}
$$


On the one hand, $m^{-1}(p)$ is nonempty of dimension equal to the dimension of $X$, since $m$ is flat and surjective. On the other hand, by projection onto $\mathcal{X}, m^{-1}(p)$ is isomorphic to the product $\mathcal{T}_{P} \times\left(\mathcal{X} \cap U_{P}\right)$, where $\mathcal{T}_{P}$ is the subgroup of $\mathcal{T}_{0}$ that acts trivially on $U_{P}$. Since $\left(\mathcal{X} \cap U_{P}\right)$ is dense in $\mathcal{X}_{P}$ we find that $\mathcal{X}_{P}$ is nonempty of dimension equal to the dimension of $X$ minus the dimension of $P$.

On the other hand, let $w$ be a point in the relative interior of $P$. Then $w$ corresponds to a cocharacter of $T$, and $w(\pi)$ specializes to a point $p$ in $U_{P}$. Projection onto $\mathcal{T}$ identifies $m^{-1}(p)$ with the mod $\pi$ reduction $\operatorname{in}_{w} X$ of $w(\pi) X$. (More formally, $\operatorname{in}_{w} X$ can be defined as the special fiber of the closure in $\mathcal{T}$ of the subscheme $w(\pi) X$ of $T$. Note that this depends only on $X$ and $T$, not on our choice of $\Sigma$.) In particular we have

$$
\operatorname{in}_{w} X \cong \mathcal{T}_{P} \times\left(\mathcal{X} \cap U_{P}\right)
$$

for any $w$ in the relative interior of $P$. We have thus shown:

Lemma 3.6. The space $\operatorname{in}_{w} X$ is a torus bundle over $\mathcal{X} \cap U_{P}$. In particular, if $C\left(\mathrm{in}_{w} X\right)$ is the set of connected components of $\left(\mathrm{in}_{w} X\right)_{\bar{k}}$, and $C\left(\mathcal{X} \cap U_{P}\right)$ is the set of connected components of $\left(\mathcal{X} \cap U_{P}\right)_{\bar{k}}$, then the maps

$$
\operatorname{in}_{w} X \cong m^{-1}(p) \rightarrow \mathcal{X} \cap U_{P}
$$

give a natural bijection of $C\left(\operatorname{in}_{w} X\right)$ with $C\left(\mathcal{X} \cap U_{P}\right)$.

We will be particularly interested in tropical pairs $(X, \mathbb{P})$ where the multiplication map $m: \mathcal{T} \times_{\mathcal{O}} \mathcal{X} \rightarrow \mathbb{P}$ is smooth. This condition is due to Tevelev.

Definition 3.7. A subvariety $X$ of $\mathcal{T}$ is schön if there exists a tropical pair $(X, \mathbb{P})$ such that the multiplication map

$$
m: \mathcal{T} \times_{\mathcal{O}} \mathcal{X} \rightarrow \mathbb{P}
$$

is smooth.

One then has ( $[\mathrm{LQ}, 6.7)$ :

Proposition 3.8. If $X$ is schön, then for any tropical pair $(X, \mathbb{P})$, the multiplication map

$$
m: \mathcal{T} \times_{\mathcal{O}} \mathcal{X} \rightarrow \mathbb{P}
$$

is smooth.

Note that if $X$ is schön then it is smooth (consider the preimage of the identity in $\mathrm{T}$ under the multiplication map.) In fact, we have:

Proposition 3.9. The following are equivalent:

(1) $X$ is schön.

(2) $\operatorname{in}_{w} X$ is smooth for all $w \in \operatorname{Trop}(X)$.

(3) For any tropical pair $(X, \mathbb{P})$, and any polyhedron $P$ in $\Sigma, \mathcal{X} \cap U_{P}$ is smooth.

Proof. Statements 2) and 3) are clearly equivalent since we have seen that $\operatorname{in}_{w} X$ is the product of a torus with $\mathcal{X} \cap U_{P}$, where $P$ is the polyhedron in $\Sigma$ that contains $w$ in its relative interior.

As for the equivalence of 1 ) and 2), fix a tropical pair $(X, \mathbb{P})$. We have seen that the fibers of the multiplication map

$$
m: \mathcal{T} \times_{\mathcal{O}} \mathcal{X} \rightarrow \mathbb{P}
$$


are isomorphic to $\operatorname{in}_{w} X$ as $w$ ranges over $\operatorname{Trop}(X)$. So 1) implies 2) is clear. For the converse, note that since $m$ is faithfully flat, to show it is smooth it suffices to show that it has smooth fibers.

It is easy to construct tropical degenerations of schön varieties $X$ in which the special fiber is a divisor with simple normal crossings. In particular we have:

Proposition 3.10 (c.f. [H] , proof of 2.4). Let $X$ be schön. There exists an integer $d$ and a tropical pair $(X, \mathbb{P})$ over $\mathcal{O}\left[\pi^{\frac{1}{d}}\right]$ such that $\bar{X}$ is smooth over $K\left[\pi^{\frac{1}{d}}\right]$, and $\mathcal{X}_{k}$ is a divisor in $\mathcal{X}$ with simple normal crossings.

Proof. Let $(X, \mathbb{P})$ be any tropical pair over $\mathcal{O}$, and let $\Sigma$ be the rational polyhedral complex corresponding to $\mathbb{P}$. By Proposition 2.3, we can find a refinement $\Sigma^{\prime}$ of $\Sigma$ and an integer $d$ such that the corresponding toric scheme $\mathbb{P}^{\prime}$ (viewed over $\mathcal{O}\left[\pi^{\frac{1}{d}}\right]$ ) has smooth general fiber, and special fiber a divisor with simple normal crossings.

Then $\left(X, \mathbb{P}^{\prime}\right)$ is also tropical, and the multiplication map

$$
m: \mathcal{T} \times_{\mathcal{O}} \mathcal{X}^{\prime} \rightarrow \mathbb{P}^{\prime}
$$

is smooth by the previous proposition. Since the special fiber of $\mathbb{P}^{\prime}$ is a divisor with simple normal crossings, so is the special fiber of $\mathcal{T} \times_{\mathcal{O}} \mathcal{X}^{\prime}$. Hence the special fiber of $\mathcal{X}^{\prime}$ is a divisor with simple normal crossings as well. Similarly, the general fiber of $\mathcal{X}^{\prime}$ is smooth because the general fiber of $\mathbb{P}^{\prime}$ is smooth.

Definition 3.11. We call a pair $(X, \mathbb{P})$ of the sort produced by Proposition 3.10 a normal crossings pair. If $(X, \mathbb{P})$ is a normal crossings pair, and $\Sigma$ is the polyhedral decomposition of $\operatorname{Trop}(X)$ corresponding to $\mathbb{P}$, we say that $\Sigma$ is a normal crossings decomposition of $\operatorname{Trop}(X)$.

Remark 3.12. In much of what follows, we will often need to attach a normal crossings pair to a schön variety $X$ over $\mathcal{O}$. To do this we may need to replace $\mathcal{O}$ with a ramified extension $\mathcal{O}\left[\pi^{\frac{1}{d}}\right]$; this is harmless and we often do so without comment.

\section{Parameterized Tropical Varieties}

In this section, given a schön subvariety $X$ of $\mathcal{T}$, we construct a natural parameterization of $\operatorname{Trop}(X)$ by a topological space $\Gamma_{X}$. This parameterization is functorial in a sense we make precise below. Moreover, we will see in the next section that the space $\Gamma_{X}$ encodes more precise information about the cohomology of $X$ than $\operatorname{Trop}(X)$ does. Our approach generalizes a construction of Speyer ( Sp2], proof of Theorem 10.8) when $X$ has dimension 1.

Suppose we have a normal crossings pair $(X, \mathbb{P})$ so $\operatorname{Supp}(\Sigma)=\operatorname{Trop}(X)$. We associate to $(X, \mathbb{P})$ a polyhedral complex $\Gamma_{(X, \mathbb{P})}$ as follows: its $k$-cells are pairs $(P, Y)$, where $P$ is a $k$-dimensional polyhedron in $\Sigma$ and $Y$ is an irreducible component of $\mathcal{X}_{P}$. The cells on the boundary of $(P, Y)$ are the cells of the form $\left(P_{i}, Y_{i}\right)$, where $P_{i}$ is a facet of $P$ and $Y_{i}$ is the irreducible component of $\mathcal{X}_{P_{i}}$ containing $Y$ (there is exactly one such irreducible component because $\mathcal{X}_{P_{i}}$ is smooth, so its irreducible components do not meet). The complex $\Gamma_{(X, \mathbb{P})}$ maps naturally to $\Sigma$ by sending $(P, Y)$ to $P$.

Proposition 4.1. The underlying topological space of $\Gamma_{(X, \mathbb{P})}$ depends only on $X$. 
Proof. Any two polyhedral decompositions of $\operatorname{Trop}(X)$ have a common refinement; we can further refine this to be a normal crossings decomposition of $\operatorname{Trop}(X)$. It thus suffices to show that if $\Sigma$ and $\Sigma^{\prime}$ are normal crossings decompositions of $\operatorname{Trop}(X)$, with associated normal crossings pairs $(X, \mathbb{P})$ and $\left(X, \mathbb{P}^{\prime}\right)$, and $\Sigma^{\prime}$ refines $\Sigma$, then the underlying topological spaces of $\Gamma_{(X, \mathbb{P})}$ and $\Gamma_{\left(X, \mathbb{P}^{\prime}\right)}$ are homeomorphic.

Since $\Sigma^{\prime}$ is a refinement of $\Sigma$, we have a map $\mathbb{P}^{\prime} \rightarrow \mathbb{P}$. Let $\mathcal{X}^{\prime}$ be the degeneration corresponding to the pair $\left(X, \mathbb{P}^{\prime}\right)$. If $P$ is a polyhedron of $\Sigma$, and $P^{\prime}$ is a polyhedron of $\Sigma^{\prime}$ contained in $P$, then this map induces a map of $\mathcal{X}_{P^{\prime}}^{\prime}$ to $\mathcal{X}_{P}$. In particular, for every pair $\left(P^{\prime}, Y^{\prime}\right)$ of $\Gamma_{X, \mathbb{P}^{\prime}}$, the image of $Y^{\prime}$ in $\mathcal{X}$ is contained in an unique irreducible component $Y$ of $\mathcal{X}_{P}$. The map taking $\left(P^{\prime}, Y^{\prime}\right)$ to $(P, Y)$ is then a map of polyhedral complexes $\Gamma_{\left(X, \mathbb{P}^{\prime}\right)} \rightarrow \Gamma_{(X, \mathbb{P})}$.

We have a commutative diagram:

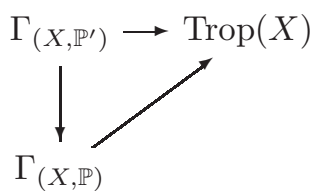

The fiber of $\pi: \Gamma_{(X, \mathbb{P})} \rightarrow \operatorname{Trop}(X)$ over a point $w$ is in canonical bijection with the set $C\left(\mathcal{X}_{P}\right)$ of (geometric) connected components of $\mathcal{X}_{P}$. Similarly, the fiber of $\pi^{\prime}: \Gamma_{\left(X, \mathbb{P}^{\prime}\right)} \rightarrow \operatorname{Trop}(X)$ over $w$ is in canonical bijection with $C\left(\mathcal{X}_{P^{\prime}}^{\prime}\right)$. By Lemma 3.6 both of these sets of connected components are in bijection with $C\left(\operatorname{in}_{w} X\right)$; in fact, we have a commutative diagram:

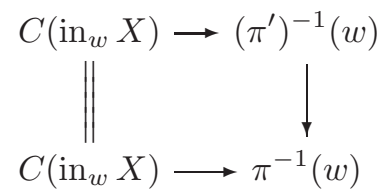

Thus the map $\Gamma_{\left(X, \mathbb{P}^{\prime}\right)} \rightarrow \Gamma_{(X, \mathbb{P})}$ is bijective, and is therefore a homeomorphism on the underlying topological spaces of $\Gamma_{\left(X, \mathbb{P}^{\prime}\right)}$ and $\Gamma_{(X, \mathbb{P})}$.

In light of this proposition, we denote by $\Gamma_{X}$ the underlying topological space of $\Gamma_{(X, \mathbb{P})}$ for any normal crossings pair $(X, \mathbb{P})$. We think of $\Gamma_{X}$, together with its natural map to $\operatorname{Trop}(X)$, as a "parameterized tropical variety." Note that $\Gamma_{X}$ inherits an integral affine structure by pullback from $\operatorname{Trop}(X)$; more precisely, for any normal crossings pair $(X, \mathbb{P})$, the map $\Gamma_{X} \rightarrow \operatorname{Trop}(X)$ is linear on any polyhedron in $\Gamma_{(X, \mathbb{P})}$.

Note that for any $w \in \operatorname{Trop}(X)$, the number of preimages of $w$ in $\Gamma_{X}$ is equal to the number of connected components of $\operatorname{in}_{w} X$. Therefore, if $\Sigma$ is a normal crossings decomposition of $\operatorname{Trop}(X)$, and $w$ is in the relative interior of a top dimensional cell $P$ of $\Sigma$, then the number of preimages of $w$ is equal to the multiplicity of $P$ in $\operatorname{Trop}(X)$. This suggests that we should give $\Gamma_{X}$ the structure of a weighted polyhedral complex by giving every polyhedron on $\Gamma_{X}$ weight one.

If we do this, then $\Gamma_{X}$ satisfies a "balancing condition" analogous to the wellknown balancing condition on $\operatorname{Trop}(X)$. Fix a normal crossings decomposition $\Sigma$ of $\operatorname{Trop}(X)$, with corresponding normal crossings pair $(X, \mathbb{P})$. Consider a polyhedron $(P, Y)$ of $\Gamma_{(X, \mathbb{P})}$ of dimension $\operatorname{dim} X-1$, and let $\left\{\left(P_{i}, Y_{i}\right)\right\}$ be the top dimensional polyhedra of $\Gamma_{(X, \mathbb{P})}$ containing $(P, Y)$.

Fix a point $w$ with rational coordinates in the relative interior of $P$, and let $V_{P}$ be the linear span, $\operatorname{Span}(P-w)$. Similarly, for each $P_{i}$, let $V_{i}$ be the positive span 
of $\operatorname{Span}^{+}\left(P_{i}-w\right)$. Then $V_{i} / V_{P}$ is a ray in $\mathbb{R}^{n} / V_{P}$; this collection of rays is the fan attached to the toric variety $\mathbb{P}_{P}$. Let $v_{i}$ be the smallest integer vector along the ray $V_{i} / V_{P}$.

Proposition 4.2. The $v_{i}$ 's satisfy the "balancing property":

$$
\sum_{\left(P_{i}, Y_{i}\right)} v_{i}=0
$$

Proof. Torus-equivariant rational functions on $\mathbb{P}_{P}$ correspond to lattice vectors $u$ in the space $\left(\mathbb{R}^{n} / V_{P}\right)^{*}$ dual to $\mathbb{R}^{n} / V_{P}$. The valuation of $u$ along the divisor of $\mathbb{P}_{P}$ corresponding to $v_{i}$ is simply $u\left(v_{i}\right)$.

Now restrict $u$ to the curve $\mathcal{X}_{P}$. For any polyhedron $P^{\prime}$ of $\Sigma$ containing $P, \mathcal{X}_{P}$ intersects the boundary divisior $\mathbb{P}_{P^{\prime}}$ in one point for each cell $\left(P_{i}, Y_{i}\right)$ of $\Gamma_{(X, \mathbb{P})}$ with $P_{i}=P^{\prime}$. The divisor of $u$ is therefore equal to

$$
\sum_{\left(P_{i}, Y_{i}\right)} u\left(v_{i}\right) Y_{i}
$$

as $\mathcal{X}_{P}$ intersects each boundary divisor $\mathbb{P}_{P_{i}}$ transversely. This divisor is a principal divisor and thus has degree zero.

We have thus attached to any schön subvariety $X$ of $\mathcal{T}$, a canonical, multiplicity free parameterization by the topological space $\Gamma_{X}$. Moreover, this construction is functorial: let $\mathcal{T}$ and $\mathcal{T}^{\prime}$ be tori over $\mathcal{O}$, and let $T$ and $T^{\prime}$ be their general fibers. Suppose we have schön subvarieties $X$ and $Y$ of $T$ and $T^{\prime}$, respectively, and a homomorphism of tori $T \rightarrow T^{\prime}$ that takes $X$ to $Y$. We then have a natural map $f: \operatorname{Trop}(X) \rightarrow \operatorname{Trop}(Y)$.

Proposition 4.3. There is a natural map $\Gamma_{X} \rightarrow \Gamma_{Y}$ that makes the diagram

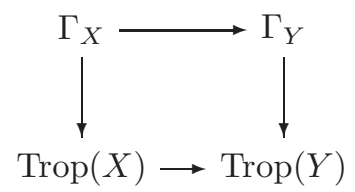

commute.

Proof. Let $\Sigma^{\prime}$ be a normal crossings decomposisition of $\operatorname{Trop}(Y)$. By proposition 3.10 we can find a normal crossings decomposition $\Sigma$ of $\operatorname{Trop}(X)$ such that the image of any cell of $\Sigma$ under the map $f$ is contained in a cell of $\Sigma^{\prime}$. Let $(X, \mathbb{P})$ and $\left(Y, \mathbb{P}^{\prime}\right)$ be the tropical pairs corresponding to $\Sigma$ and $\Sigma^{\prime}$, and let $\mathcal{X}$ and $\mathcal{Y}$ denote the associated tropical degenerations. Since each cell of $\Sigma$ maps into a cell of $\Sigma^{\prime}$, we obtain a map from $\mathcal{X}$ to $\mathcal{Y}$ extending the map $X \rightarrow Y$.

Now let $P$ be a polyhedron in $\Sigma$, and $P^{\prime}$ be the polyhedron of $\Sigma^{\prime}$ containing the image of $P$. Then our map $\mathcal{X} \rightarrow \mathcal{Y}$ induces a map $\mathcal{X}_{P} \rightarrow \mathcal{Y}_{P^{\prime}}$.

If $\left(P, X_{i}\right)$ is a polyhedron of $\Gamma_{(X, \mathbb{P})}$, then by definition $X_{i}$ is a connected component of $\mathcal{X}_{P}$. The image of $X_{i}$ in $\mathcal{Y}_{P^{\prime}}$ is contained in a connected component $Y_{i}$ of $\mathcal{Y}_{P^{\prime}}$. We can thus construct a map of polyhedral complexes

$$
\Gamma_{(X, \mathbb{P})} \rightarrow \Gamma_{\left(Y, \mathbb{P}^{\prime}\right)}
$$

that maps $\left(P, X_{i}\right)$ to $\left(P^{\prime}, Y_{i}\right)$ by the map $P \rightarrow P^{\prime}$. The induced map $\Gamma_{X} \rightarrow$ $\Gamma_{Y}$ on underlying topological spaces is clearly continuous and makes the diagram commute. 
To see that it is independent of choices, let $\pi_{X}$ and $\pi_{Y}$ be the projections of $\Gamma_{X}$ and $\Gamma_{Y}$ to $\operatorname{Trop}(X)$ and $\operatorname{Trop}(Y)$ respectively. We then have canonical bijections between $\pi_{X}^{-1}(w)$ and $C\left(\operatorname{in}_{w} X\right)$, and between $\pi_{Y}^{-1}(f(w))$ and $C\left(\operatorname{in}_{f(w)} Y\right)$. The map $X \rightarrow Y$ induces a natural map $\operatorname{in}_{w} X \rightarrow \operatorname{in}_{f(w)} Y$, and the diagram

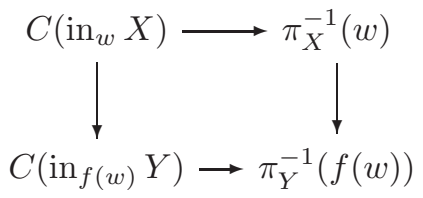

commutes. As the left hand side is independent of the choices of $\Sigma$ and $\Sigma^{\prime}$, the result follows.

Remark 4.4. Although Proposition 4.3 is stated for maps $X \rightarrow Y$ that are monomial morphisms (i.e., that arise from morphisms of the ambient tori), we can avoid this issue if $X$ and $Y$ are intrinsically embedded. Recall that $X$ is very affine if it can be embedded as a closed subscheme of a torus $T$. In this case (c.f. T, section 3 ) there is an intrinsic torus $T_{X}$ associated to $X$ a canonical embedding of $X$ in $T_{X}$. Moreover, if $X$ and $Y$ are very affine and $f: X \rightarrow Y$ is a morphism, there is a morphism of tori $T_{X} \rightarrow T_{Y}$ that induces $f$.

We also record, for later use, the following result relating the cohomology of $\Gamma_{X}$ to that of $\operatorname{Trop}(X)$ :

Lemma 4.5. Let $X$ be schön, and let $\Sigma$ be a normal crossings decomposition of $\operatorname{Trop}(X)$. Suppose that for each polyhedron $P$ in $\Sigma, \mathcal{X}_{P}$ is either connected or has dimension zero. Then the natural map

$$
H^{r}(\operatorname{Trop}(X), \mathbb{Z}) \rightarrow H^{r}\left(\Gamma_{X}, \mathbb{Z}\right)
$$

is an isomorphism for $0 \leq r<\operatorname{dim} X$, and an injection for $r=\operatorname{dim} X$.

Proof. Let $(X, \mathbb{P})$ be the normal crossings pair attached to $\Sigma$, so that $\Gamma_{(X, \mathbb{P})}$ is a triangulation of $\Gamma$. The polyhedra $P$ in $\Gamma_{(X, \mathbb{P})}$ with $\operatorname{dim} P<\operatorname{dim} X$ are, by construction, in bijection with the polyhedra in $\Sigma$ with $\operatorname{dim} P<\operatorname{dim} X$. Thus $\Gamma_{(X, \mathbb{P})}$ is obtained from $\Sigma$ by adding additional top-dimensional cells; the result follows immediately.

\section{Weight filtrations and the Weight speCtral SeQuence}

Our goal will be to relate the combinatorial structure of $\Gamma_{X}$ to geometric invariants of $X$. The invariants that appear arise from Deligne's theory of weights, which we now summarize. Recall (c.f. [D, 1.2) that if $F$ is a finite field of order $q$, a continuous $l$-adic representation $\rho$ of $\operatorname{Gal}\left(F^{\mathrm{sep}} / F\right)$ has weight $r$ if all the eigenvalues of the geometric Frobenius of $F$ are algebraic integers $\alpha$, all of whose Galois conjugates have complex absolute value equal to $q^{r / 2}$. If $A$ is a finitely generated $\mathbb{Z}$-algebra, an étale sheaf $\mathcal{F}$ on $\operatorname{Spec} A$ has weight $r$ if for each closed point $s$ of Spec $A$, the stalk $\mathcal{F}_{s}$ has weight $r$ when considered as a $\operatorname{Gal}\left(k(s)^{\operatorname{sep}} / k(s)\right)$-module.

Following Ito $(\mathbb{I}, 2.2)$, we extend this definition to the case where $F$ is a purely inseparable extension of a finitely generated extension of $\mathbb{F}_{p}$ or $\mathbb{Q}$. For such $F$, one can find a finitely generated $\mathbb{Z}$-subalgebra $A$ of $F$ such that $F$ is a purely inseparable extension of the field of fractions of $A$.

In this setting, a representation $\rho$ of $\operatorname{Gal}\left(F^{\text {sep }} / F\right)$ has weight $r$ if there is an open subset $U$ of Spec $A$, and a smooth $\mathcal{F}$ on $U$ of weight $r$, such that $\rho$ arises from $\mathcal{F}$ by 
pullback to $\operatorname{Spec} F$. The Weil conjectures imply that for any proper smooth variety $X$ over $F$, and any $l$ prime to the characteristic of $F, H_{\text {ét }}^{r}\left(X_{F^{\text {sep }}}, \mathbb{Q}_{l}\right)$ has weight $r$.

We henceforth assume that the residue field $k$ of $\mathcal{O}$ is a purely inseparable extension of a finitely generated extension of $\mathbb{F}_{p}$ or $\mathbb{Q}$. We also fix an $l$ prime to the characteristic of $k$.

Let $G$ be the absolute Galois group of the field $K$. Then $G$ admits a surjection $G \rightarrow \operatorname{Gal}\left(k^{\text {sep }} / k\right)$, whose kernel is the inertia group $I_{K}$ of $K$. If $M$ is a $G$-module on which $I$ acts through a finite quotient, there is a finite index subgroup $H$ in $G$ such that $H \cap I$ acts trivially on $M$. Thus $\operatorname{Gal}\left(k^{\text {sep }} / k^{\prime}\right)$ acts on $M$ for some finite extension $k^{\prime}$ of $k$. We say $M$ is pure of weight $r$ if it has weight $r$ as a $\operatorname{Gal}\left(k^{\text {sep }} / k^{\prime}\right)$-module. Note that this is independent of $k^{\prime}$.

The étale cohomology of a variety over $K$ with semistable reduction has a filtration by subquotients which are pure in the above sense. More precisely, let $\mathcal{X}$ be a proper scheme over $\mathcal{O}$, of relative dimension $n$, whose fiber $X_{K}$ over Spec $K$ is smooth and whose fiber $\mathcal{X}_{k}$ over Spec $k$ is a divisor with simple normal crossings. Then the Rapoport-Zink weight spectral sequence relates the étale cohomology of $X_{K^{\text {sep }}}$ to the geometry of the special fiber $\mathcal{X}_{k}$. More precisely, let $\mathcal{X}_{k^{\text {sep }}}^{(r)}$ denote the disjoint union of $(r+1)$-fold intersections of irreducible components of $\mathcal{X}_{k^{\text {sep }}}$; it is smooth of dimension $n-r$ over $k^{\text {sep }}$. We then have:

Theorem 5.1 ([RZ, Satz 2.10; see also [1] $)$. There is a spectral sequence:

$$
E_{1}^{-r, w+r}=\bigoplus_{s \geq \max (0,-r)} H_{\text {ét }}^{w-r-2 s}\left(\mathcal{X}_{k^{\text {sep }}}^{(2 s+r)}, \mathbb{Q}_{l}(-r-s)\right) \Rightarrow H_{\text {ét }}^{w}\left(X_{K^{\mathrm{sep}}}, \mathbb{Q}_{l}\right) .
$$

Here $\mathbb{Q}_{l}(n)$ is the $n$th "Tate twist" of the constant sheaf $\mathbb{Q}_{l}$; that is, it is the tensor product of $\mathbb{Q}_{l}$ with the $n$th tensor power of the sheaf $\mathbb{Z}_{l}(1)$, where $\mathbb{Z}_{l}(1)$ is the inverse limit of the sheaves $\mu_{l^{n}}$ of $l$-power roots of unity. Note that $\mathbb{Z}_{l}(1)$ is pure of weight -2 .

The boundary maps of this spectral sequence are completely explicit, and can be described as follows: up to sign, they are direct sums of restriction maps

$$
H_{\text {ét }}^{i}\left(Y, \mathbb{Q}_{l}(-m)\right) \rightarrow H_{\text {èt }}^{i}\left(Y^{\prime}, \mathbb{Q}_{l}(-m)\right)
$$

where $Y$ is an irreducible component of $\mathcal{X}_{k^{\mathrm{sep}}}^{(j)}$ and $Y^{\prime}$ is an irreducible component of $\mathcal{X}_{k^{\mathrm{sep}}}^{(j+1)}$ contained in $Y$, or Gysin maps

$$
H_{\text {ét }}^{i}\left(Y^{\prime}, \mathbb{Q}_{l}(-m)\right) \rightarrow H_{\text {ét }}^{i+2}\left(Y, \mathbb{Q}_{l}(-m+1)\right)
$$

where $Y$ and $Y^{\prime}$ are as above.

More precisely, each term $E_{1}^{p, q}$ is a direct sum of terms of the form $H_{\text {ett }}^{i}\left(Y, \mathbb{Q}_{l}(-m)\right)$ for some irreducible component $Y$ of $\mathcal{X}_{k^{s e p}}^{(j)}$. If $Y^{\prime}$ is an irreducible component of $\mathcal{X}_{k^{\text {sep }}}^{(j+1)}$, then we have:

- Whenever $H_{\text {ét }}^{i}\left(Y, \mathbb{Q}_{l}(-m)\right)$ is a direct summand of $E_{1}^{p, q}$, and $H_{\text {ét }}^{i}\left(Y^{\prime}, \mathbb{Q}_{l}(-m)\right)$ is a direct summand of $E_{1}^{p+1, q}$, then the corresponding direct summand of the boundary map $E_{1}^{p, q} \rightarrow E_{1}^{p+1, q}$ is (up to sign) the restriction

$$
H_{\text {ét }}^{i}\left(Y, \mathbb{Q}_{l}(-m)\right) \rightarrow H_{\text {ét }}^{i}\left(Y^{\prime}, \mathbb{Q}_{l}(-m)\right) .
$$

- Whenever $H_{\text {êt }}^{i}\left(Y^{\prime}, \mathbb{Q}_{l}(-m)\right)$ is a direct summand of $E_{1}^{p, q}$, and $H_{\text {êt }}^{i}\left(Y, \mathbb{Q}_{l}(-m+\right.$ $1))$ is a direct summand of $E_{1}^{p+1, q}$, then the corresponding direct summand 
of the boundary map $E_{1}^{p, q} \rightarrow E_{1}^{p+1, q}$ is (up to sign) the Gysin map

$$
H_{\text {ét }}^{i}\left(Y^{\prime}, \mathbb{Q}_{l}(-m)\right) \rightarrow H_{\text {ét }}^{i+2}\left(Y, \mathbb{Q}_{l}(-m+1)\right) .
$$

We refer the reader to example 7.5 for a description of the weight spectral sequence in the case when $X$ is a smooth curve.

Note that the term $E_{1}^{-r, w+r}$ of the weight spectral sequence is pure of weight $w+r$. As the only map between $\mathbb{Q}_{l}$-sheaves that are pure of different weights is the zero map, this implies that the weight spectral sequence degenerates at $E_{2}$. Moreover, the successive quotients of the filtration on $H_{\text {et }}^{*}\left(X_{K^{\text {sep }}}, \mathbb{Q}_{l}\right)$ induced by the weight spectral sequence are pure. The filtration arising in this way is called the weight filtration on $H_{\text {êt }}^{*}\left(X_{K^{\text {sep }}}, \mathbb{Q}_{l}\right)$.

We say a $G$-module $M$ is mixed if $M$ admits an increasing $G$-stable filtration

$$
\cdots \subset W_{r} M \subset W_{r+1} M \subset \ldots
$$

such that $W_{r} M / W_{r-1} M$ has weight $r$ for all $r$. (Such a filtration, if it exists, will be unique.) We say $M$ is mixed of weights between $r$ and $r^{\prime}$ if $M$ is mixed and the quotients $W_{i} M / W_{i-1} M$ are nonzero only when $r \leq i \leq r^{\prime}$. The above result shows that the cohomology of any scheme over $K$ with semistable reduction is mixed. More generally, one has:

Theorem 5.2. (c.f. I], 2.3) Let $X$ be a smooth, proper $n$-dimensional variety over $K$. Then $H_{\text {ét }}^{r}\left(X_{K^{\text {sep }}}, \mathbb{Q}_{l}\right)$ is mixed of weights between $\max (0,2 r-2 n)$ and $\min (2 n, 2 r)$.

Proof. If $X$ has strictly semistable reduction, i.e., $X$ is isomorphic to the general fiber of a scheme $\mathcal{X}$ that is proper over $\mathcal{O}$, and whose special fiber is a divisor with simple normal crossings, then this follows from the weight spectral sequence. The general case follows by de Jong's theory of alterations [d].

Proposition 5.3. Let $X$ be a smooth $n$-dimensional variety over $K$, and $\bar{X}$ a compactification of $X$ such that $\bar{X}-X$ is a divisor with simple normal crossings. Then for $r \leq n, H_{\text {ét }}^{r}\left(X_{K^{\text {sep }}}, \mathbb{Q}_{l}\right)$ is mixed of weights between 0 and $2 r$, and the natural map

$$
W_{0} H_{\text {ét }}^{r}\left(\bar{X}_{K^{\text {sep }}}, \mathbb{Q}_{l}\right) \rightarrow W_{0} H_{\text {ét }}^{r}\left(X_{K^{\text {sep }}}, \mathbb{Q}_{l}\right)
$$

is an isomorphism.

Proof. Let $D$ be the divisor $\bar{X} \backslash X$, and let $\bar{D}_{1}, \ldots, \bar{D}_{r}$ be its irreducible components. Let $X_{i}$ be the open subset $X \backslash\left\{\bar{D}_{1} \cup \cdots \cup \bar{D}_{i}\right\}$. We proceed by induction on $i$; the case $i=0$ is clear.

Suppose the proposition is true for $i$. Define

$$
D_{i}=\bar{D}_{i+1} \backslash\left\{\bar{D}_{1} \cup \cdots \cup \bar{D}_{i}\right\},
$$

so that $X_{i} \backslash X_{i+1}=D_{i}$. By the inductive hypothesis the spaces $H_{\text {ét }}^{r}\left(\left(X_{i}\right)_{L}, \mathbb{Q}_{l}\right)$ and $H_{\text {ét }}^{r}\left(\left(D_{i}\right)_{L}, \mathbb{Q}_{l}\right)$ are mixed of weights between 0 and $2 r$ for $r \leq n$. We have a Gysin sequence:

$$
\begin{aligned}
& H_{\text {ét }}^{r-2}\left(\left(D_{i}\right)_{L}, \mathbb{Q}_{l}(-1)\right) \rightarrow H_{\text {ét }}^{r}\left(\left(X_{i}\right)_{L}, \mathbb{Q}_{l}\right) \rightarrow H_{\text {ét }}^{r}\left(\left(X_{i+1}\right)_{L}, \mathbb{Q}_{l}\right) \rightarrow \\
& H_{\text {ét }}^{r-1}\left(\left(D_{i}\right)_{L}, \mathbb{Q}_{l}(-1)\right) \rightarrow \quad \ldots,
\end{aligned}
$$

and the first and last terms are mixed of weights between 2 and $2 r$. It follows that $H_{\text {ét }}^{r}\left(\left(X_{i+1}\right)_{L}, \mathbb{Q}_{l}\right)$ is mixed of weights between 0 and $2 r$ as required. We also obtain an isomorphism

$$
W_{0} H_{\text {ét }}^{r}\left(\left(X_{i}\right)_{L}, \mathbb{Q}_{l}\right) \cong W_{0} H_{\text {ét }}^{r}\left(\left(X_{i+1}\right)_{L}, \mathbb{Q}_{l}\right)
$$


and hence by induction the desired isomorphism

$$
W_{0} H_{\text {ét }}^{r}\left(\bar{X}_{L}, \mathbb{Q}_{l}\right) \cong W_{0} H_{\text {ét }}^{r}\left(X_{L}, \mathbb{Q}_{l}\right) \text {. }
$$

\section{COHOMOLOGY OF SCHÖN VARIETIES}

The control that tropical geometry gives over the degenerations of schön subvarieties $X$ of $T$ has significant consequences on the level of cohomology. In particular the theory of vanishing cycles allows one to relate the étale cohomology of a nice tropical compactification of $X$ to that of its tropical degeneration. When the degeneration is a divisor with simple normal crossings, this relationship is given by the Rapoport-Zink weight spectral sequence.

We apply this sequence in the setting of tropical geometry. Let $X$ be schön. By Proposition 3.10 there is a polyhedral complex $\Sigma$, with support equal to $\operatorname{Trop}(X)$ and corresponding toric scheme $\mathbb{P}$, such that the pair $(X, \mathbb{P})$ is tropical, the corresponding compactification $\bar{X}$ of $X$ is smooth with simple normal crossings boundary, and the special fiber of the corresponding tropical degeneration $\mathcal{X}$ is a divisor with simple normal crossings.

The polyhedral complex $\Gamma_{(X, \mathbb{P})}$ encodes the combinatorics of the special fiber $\mathcal{X}_{k^{\text {sep }}}$. In particular $\mathcal{X}_{k^{\text {sep }}}$ is a union of smooth connected varieties $\mathcal{X}_{v}$, where $v$ runs over the vertices of $\Gamma_{(X, \mathbb{P})}$. The varieties $\mathcal{X}_{v_{1}}, \ldots, \mathcal{X}_{v_{r}}$ meet if and only if $v_{1}, \ldots, v_{r}$ are the vertices of a polyhedron in $\Gamma_{(X, \mathbb{P})}$. [Note that since $\mathcal{X}_{k}$ is a simple normal crossings divisor, if $Y_{0}, \ldots, Y_{r}$ intersect in codimension $r$ then they are the only irreducible components of $\mathcal{X}_{k^{\text {sep }}}$ containing their intersection.]

We have a natural map $\Gamma_{(X, \mathbb{P})} \rightarrow \Sigma$. Since $\Sigma$ is a triangulation of $\operatorname{Trop}(X)$, and $\Gamma_{(X, \mathbb{P})}$ is a triangulation of $\Gamma_{X}$, this induces a natural map

$$
H^{r}\left(\operatorname{Trop} X, \mathbb{Q}_{l}\right) \rightarrow H^{r}\left(\Gamma_{X}, \mathbb{Q}_{l}\right) .
$$

By the proof of Lemma 4.5 this map is an isomorphism if $\mathcal{X}_{P}$ is connected for every polyhedron $P$ in $\Sigma$, or (equivalently) if $\operatorname{in}_{w} X$ is connected for every $w$ in $\operatorname{Trop}(X)$.

Theorem 6.1. There is a natural isomorphism

$$
H^{r}\left(\Gamma_{X}, \mathbb{Q}_{l}\right) \cong W_{0} H_{\text {ét }}^{r}\left(\bar{X}_{K^{\text {sep }}}, \mathbb{Q}_{l}\right),
$$

and hence a natural map

$$
H^{r}\left(\operatorname{Trop}(X), \mathbb{Q}_{l}\right) \rightarrow W_{0} H_{\text {ét }}^{r}\left(\bar{X}_{K^{\text {sep }}}, \mathbb{Q}_{l}\right) .
$$

This map is an isomorphism if $\mathcal{X}_{P}$ is connected for every polyhedron $P$ in $\Sigma$.

Proof. The bottom nonzero row of the $E_{1}$ term of the Rapoport-Zink spectral sequence (i.e., the $w=-r$ row) is the complex:

$$
H_{\text {ét }}^{0}\left(\mathcal{X}_{k^{\text {sep }}}^{(0)}, \mathbb{Q}_{l}\right) \rightarrow H_{\text {ét }}^{0}\left(\mathcal{X}_{k^{\text {sep }}}^{(1)}, \mathbb{Q}_{l}\right) \rightarrow H_{\text {ét }}^{0}\left(\mathcal{X}_{k^{\text {sep }}}^{(2)}, \mathbb{Q}_{l}\right) \rightarrow \ldots
$$

in which the horizontal maps are restriction maps. This is simply the coboundary complex of the polyhedral complex formed by the bounded cells of $\Gamma_{(X, \mathbb{P})}$. This polyhedral complex is homotopy equivalent to $\Gamma_{(X, \mathbb{P})}$. We thus have a natural isomorphism

$$
E_{2}^{r, 0} \cong H^{r}\left(\Gamma_{X}, \mathbb{Q}_{l}\right)
$$


Remark 6.2. Theorem 6.1 shows in particular that the space $W_{0} H_{\text {ét }}^{r}\left(\bar{X}_{K^{\mathrm{sep}}}, \mathbb{Q}_{l}\right)$, which a priori depends on $\bar{X}$ and thus a choice of $\Sigma$, in fact depends only on $X$ and is independent of $\Sigma$. Proposition 5.3 establishes this directly on the level of cohomology.

The above results allow us to translate results about the cohomology of complete intersections in toric varieties into results about their tropicalizations. For instance:

Corollary 6.3. Let $X$ be a schön subvariety of $T$, and $\mathbb{P}_{K}$ a smooth projective toric variety of $T$ such that:

(1) the closure $Z$ of $X$ in $\mathbb{P}_{K}$ is a smooth complete intersection of ample divisors, and

(2) the boundary $Z \backslash X$ is a divisor with simple normal crossings.

Then $H^{r}\left(\Gamma_{X}, \mathbb{Q}_{l}\right)=0$ for $1 \leq r<\operatorname{dim} X$.

Proof. By Proposition 3.10 and Theorem 6.1 there is a tropical pair $\left(X, \mathbb{P}^{\prime}\right)$, with corresponding compactification $\bar{X}$ of $X$, such that $H^{r}\left(\Gamma_{X}, \mathbb{Q}_{l}\right)$ is isomorphic to $W_{0} H_{\text {ét }}^{r}\left(\bar{X}_{K^{\text {sep }}}, \mathbb{Q}_{l}\right)$. By Proposition 5.3 the latter is isomorphic to $W_{0} H_{\text {ét }}^{r}\left(Z_{K^{\text {sep }}}, \mathbb{Q}_{l}\right)$.

Since $Z$ is a complete intersection in $\mathbb{P}_{K}$, the Lefschetz hyperplane theorem shows that for $r<\operatorname{dim} X$, the restriction map

$$
H_{\text {ét }}^{r}\left(\mathbb{P}_{K^{\text {sep }}}, \mathbb{Q}_{l}\right) \rightarrow H_{\text {ét }}^{r}\left(Z_{K^{\mathrm{sep}}}, \mathbb{Q}_{l}\right)
$$

is an isomorphism. But $\mathbb{P}_{K}$ is a smooth toric variety, and hence has good reduction. The weight spectral sequence thus shows that $W_{0} H_{\text {ét }}^{r}\left(\mathbb{P}_{K^{\text {sep }}}, \mathbb{Q}_{l}\right)=0$ for $r>0$.

Under more restrictive hypotheses on $X$, we can turn the above result into a result about the cohomology of $\operatorname{Trop}(X)$. This will be the main goal of section 8

\section{Monodromy}

In many situations, the weight filtration has an alternative interpretation in terms of monodromy. Let $X$ be a variety over $K$, and consider the base change $X_{K^{\text {sep }}}$ of $X$ to $K^{\text {sep }}$. The group $\operatorname{Gal}\left(K^{\text {sep }} / K\right)$ admits a map to $\operatorname{Gal}\left(k^{\text {sep }} / k\right)$; the kernel is the inertia group $I_{K}$. The group $I_{K}$ is profinite; if $l$ is prime to the characteristic of $k$ then the pro- $l$ part $I_{K}^{(l)}$ of $I_{K}$ is isomorphic to $\mathbb{Z}_{l}(1)$. (The Tate twist here refers to the fact that the quotient $\operatorname{Gal}\left(k^{\text {sep }} / k\right)$ acts on $I_{K}^{(l)}$ by conjugation in the same way that it acts on the inverse limit of the roots of unity $\mu_{l^{n}}$.

The group $\operatorname{Gal}\left(K^{\mathrm{sep}} / K\right)$ acts on the étale cohomology $H_{\text {ét }}^{i}\left(X_{K^{\text {sep }}}, \mathbb{Q}_{l}\right)$ for any prime $l$. This action is quasi-unipotent, i.e. a subgroup of $H$ of $I_{K}$ of finite index acts unipotently on $H_{\text {ét }}^{i}\left(X_{K^{\mathrm{sep}}}, \mathbb{Q}_{l}\right)$. (And thus the action of $H$ factors through $I_{K}^{(l)}$.) In particular there is a nilpotent map

$$
N: H_{\text {ét }}^{i}\left(X_{K^{\mathrm{sep}}}, \mathbb{Q}_{l}\right) \rightarrow H_{\text {ét }}^{i}\left(X_{K^{\mathrm{sep}},}, \mathbb{Q}_{l}(-1)\right)
$$

called the monodromy operator such that for all $\sigma \in H, \sigma$ acts on $H_{\text {êt }}^{i}\left(X_{K^{\text {sep }}}, \mathbb{Q}_{l}\right)$ by $\exp \left(t_{l}(\sigma) N\right)$, where $t_{l}$ is the map $I_{K} \rightarrow I_{K}^{(l)} \cong \mathbb{Z}_{l}(1)$.

Now, if $V$ is any finite dimensional vector space, with a nilpotent endomorphism $N$ such that $N^{r}=0$, then there is a unique increasing filtration $\left\{V_{i}\right\}$ on $V$ such that:

- $V_{r}=V$,

- $V_{-r}=0$,

- $N$ maps $V_{i}$ to $V_{i-2}$, and 
- $N^{i}$ induces an isomorphism $V_{i} / V_{i-1} \rightarrow V_{-i} / V_{-i-1}$.

(see [D] I, 1.7.2 for details.) We thus obtain a natural filtration, called the monodromy filtration, on $H_{\text {ét }}^{i}\left(X_{K^{\mathrm{sep}}}, \mathbb{Q}_{l}\right)$.

Remark 7.1. If $V$ consists of a single Jordan block of dimension $r$, one sees easily that $V_{i} / V_{i-1}$ is one-dimensional for $i \in\{r-1, r-3, \ldots,-r+1\}$, and zero otherwise. Moreover, $V_{r-1-2 k}$ is the image of $N^{k}$ for $0 \leq k \leq r-1$. It is thus straightforward to read off the filtration coming from an arbitrary $V$ and $N$ from a Jordan normal form for $N$. The filtration is independent of choices, even though the Jordan normal form of $N$ is not.

When $X$ has a semistable model, one can read the monodromy action on $X$ off of the weight spectral sequence $E^{p, q}$. More precisely, there is a monodromy operator $N: E_{1}^{p, q} \rightarrow E_{1}^{p+2, q-2}(-1)$, which converges to the the monodromy operator $N$ on $H_{\text {ét }}^{i}\left(X_{K^{\text {sep }}}, \mathbb{Q}_{l}\right)$. It is easily described: if $H_{\text {ét }}^{i}\left(Y, \mathbb{Q}_{l}(-m)\right)$ occurs as a direct summand of $E_{1}^{p, q}$, and $H_{\text {ét }}^{i}\left(Y, \mathbb{Q}_{l}(-m+1)\right)$ occurs as a direct summand of $E_{1}^{p+2, q-2}$, then the corresponding direct summand of $N$ is the identity

$$
H_{\text {ét }}^{i}\left(Y, \mathbb{Q}_{l}(-m)\right) \rightarrow H_{\text {ét }}^{i}\left(Y, \mathbb{Q}_{l}(-m+1)\right)(-1) .
$$

All other direct summands of $N$ are the zero map.

The following conjecture (the "weight-monodromy conjecture") relates the weight filtration to the monodromy filtration in this situation:

Conjecture 7.2. The top nonzero power of the monodromy operator:

$$
N^{r}: E_{2}^{-r, w+r} \rightarrow E_{2}^{r, w-r}
$$

is an isomorphism for all $r, w$. In particular the weight filtration on $H_{\text {ét }}^{i}\left(X_{K^{\mathrm{sep}}}, \mathbb{Q}_{l}\right)$ $E$ coincides (up to a shift in degree) with the monodromy filtration; that is,

$$
H_{\text {ét }}^{w}\left(X_{K^{\text {sep }}}, \mathbb{Q}_{l}\right)_{-r} / H_{\text {ét }}^{w}\left(X_{K^{\text {sep }}}, \mathbb{Q}_{l}\right)_{-r-1} \cong W_{w-r} H_{\text {ét }}^{w}\left(X_{K^{\text {sep }}}, \mathbb{Q}_{l}\right) \text {. }
$$

The weight-monodromy conjecture is well-known to hold for curves and surfaces. If $\mathcal{O}$ is an equal characteristic discrete valuation ring, it is a difficult theorem of Ito ([I], Theorem 1.1). It is open in general when $\mathcal{O}$ has mixed characteristic.

For the remainder of this section we assume we are in a situation where Conjecture 7.2 holds. The following result, due to Speyer ([Sp2], Theorem 10.8) for curves, follows immediately:

Corollary 7.3. Let $b_{r}\left(\Gamma_{X}\right)$ and $b_{r}(X)$ denote the rth Betti numbers of $\Gamma_{X}$ and $X$, respectively. Then we have:

$$
b_{r}\left(\Gamma_{X}\right) \leq \frac{1}{r+1} b_{r}(X)
$$

Proof. Theorem 6.1 together with the weight-monodromy conjecture, shows that $b_{r}\left(\Gamma_{X}\right)$ is the dimension of $H_{\text {ét }}^{r}\left(\bar{X}_{K^{\text {sep }}}, \mathbb{Q}_{l}\right)_{-r}$. The dimension of this piece of the monodromy filtration counts the number of Jordan blocks of size $r+1$ in a Jordan normal form for $N$ acting on $H_{\text {ét }}^{r}\left(\bar{X}_{K^{\text {sep }}}, \mathbb{Q}_{l}\right)$. In particular the dimension of the latter is at least $r+1$ times the dimension of the former.

Suppose $X_{K}$ is an $n$-dimensional variety. There is a geometric interpretation of the action of the $n$th power of the monodromy operator on the middle-dimensional cohomology. 
Proposition 7.4. The dth power of the monodromy map acting on middle cohomology,

$$
N^{d}: H_{\text {ét }}^{d}\left(X_{K^{\mathrm{sep}}}, \mathbb{Q}_{l}\right)_{d} / H_{\text {ét }}^{d}\left(X_{K^{\mathrm{sep}}}, \mathbb{Q}_{l}\right)_{d-1} \rightarrow H_{\text {ét }}^{d}\left(X_{K^{\mathrm{sep}}}, \mathbb{Q}_{l}\right)_{-d}(-d) .
$$

is the map

$$
H_{d}\left(\Gamma_{(X, \mathbb{P})}, \mathbb{Q}_{l}\right)(-d) \rightarrow H^{d}\left(\Gamma_{(X, \mathbb{P})}, \mathbb{Q}_{l}\right)(-d)
$$

induced from the "volume pairing" on the parameterizing complex $\Gamma_{(X, \mathbb{P})}$ which takes a pair of (integral) d-dimensional cycles to the (oriented) lattice volume of their intersection.

Proof. The term $H_{\text {ét }}^{d}\left(X_{K^{\text {sep }}}, \mathbb{Q}_{l}\right)_{d} / H_{\text {ét }}^{d}\left(X_{K^{\text {sep }}}, \mathbb{Q}_{l}\right)_{d-1}$ is computed by the $(-d, d)$ entry in the Rapoport-Zink spectral sequence. The $d$ th row is:

$$
H_{\text {ét }}^{0}\left(\mathcal{X}_{k^{\text {sep }}}^{(d)}, \mathbb{Q}_{l}\right)(-d) \rightarrow H_{\text {ét }}^{2}\left(\mathcal{X}_{k^{\text {sep }}}^{(d-1)}, \mathbb{Q}_{l}\right)(-d+1) \rightarrow \cdots \rightarrow H_{\text {ét }}^{2 d}\left(\mathcal{X}_{k^{\text {sep }}}^{(0)}, \mathbb{Q}_{l}\right)
$$

where the horizontal map is the Gysin map of $\mathcal{X}^{(k)} \rightarrow \mathcal{X}^{(k-1)}$. Since each component of $\mathcal{X}^{(k)}$ is an $(d-k)$-dimensional smooth variety, this is the chain complex formed by the bounded cells of $\Gamma_{(X, \mathbb{P})}$. Consequently, $E_{2}^{-k, d} \cong H_{k}\left(\Gamma, \mathbb{Q}_{l}\right)(-k)$. Now,

$$
N^{d}: E_{2}^{-d, d} \cong H_{d}\left(\Gamma, \mathbb{Q}_{l}\right)(-d) \rightarrow E_{2}^{0,0}(-d) \cong H^{d}\left(\Gamma_{(X, \mathbb{P})}, \mathbb{Q}_{l}\right)(-d)
$$

is induced from the identity map on $H_{\text {ét }}^{0}\left(\mathcal{X}_{k^{\text {sep }}}^{(d)}, \mathbb{Q}_{l}\right)$. In the language of homology and cohomology of $\Gamma_{(X, \mathbb{P})}$, it comes from the map $C_{d}\left(\Gamma_{(X, \mathbb{P})}\right) \rightarrow C^{d}\left(\Gamma_{(X, \mathbb{P})}\right)$ taking a simplex $F$ to the cocycle $\delta_{F}: C^{d}\left(\Gamma_{(X, \mathbb{P})}\right) \rightarrow \mathbb{Z}$ that is the indicator function of $F$. Consequently, if we view $N^{d}$ as a bilinear pairing on $H_{d}\left(\Gamma, \mathbb{Q}_{l}\right)$, it is the volume pairing as every bounded top-dimensional cell of $\Gamma_{(X, \mathbb{P})}$ has volume 1.

Example 7.5. Suppose that $X_{K}$ is a curve of genus $g$. Then $\mathcal{X}_{k^{\text {sep }}}^{(0)}$ is the normalization of $\mathcal{X}_{k^{\text {sep }}}$; it is a disjoint union of smooth curves $C_{i}$ of genus $g_{i}$. On the other hand, $\mathcal{X}_{k^{\text {sep }}}^{(1)}$ is the set of singular points of $\mathcal{X}_{k^{\text {sep }}}$; each such point lies on exactly two of the $C_{i}$. The corresponding weight spectral sequence is nonzero only for $-1 \leq r \leq 1$ and $0 \leq w+r \leq 2$; it looks like:

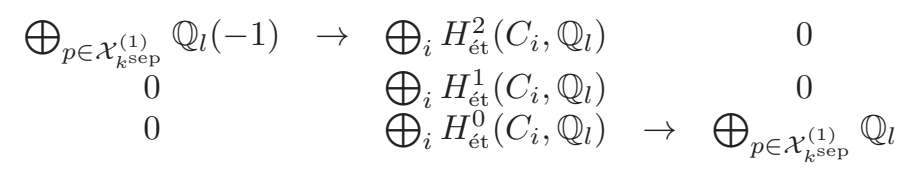

The sequence clearly degenerates at $E_{2}$. The monodromy operator $N$ is nonzero only from $E_{1}^{-1,2}$ to $E_{1}^{1,0}(-1)$; it is simply the identity map on

$$
\bigoplus_{p \in \mathcal{X}_{k^{\text {sep }}}^{(1)}} \mathbb{Q}_{l}(-1)
$$

We thus find that the middle quotient of the monodromy filtration on $H_{\text {êt }}^{1}\left(X_{K_{\text {sep }}}, \mathbb{Q}_{l}\right)$ is isomorphic to the direct sum of $H_{\text {êt }}^{1}\left(C_{i}, \mathbb{Q}_{l}\right)$, whereas the top and bottom quotients are isomorphic to $H_{1}\left(\Gamma, \mathbb{Q}_{l}\right)$, (resp. $\left.H^{1}\left(\Gamma, \mathbb{Q}_{l}\right)\right)$ where $\Gamma$ is the dual graph of $\mathcal{X}_{k^{\text {sep }}}$. As above, the map $N: H_{1}\left(\Gamma, \mathbb{Q}_{l}\right) \rightarrow H^{1}\left(\Gamma, \mathbb{Q}_{l}\right)$ can be interpreted as the length pairing on $H_{1}\left(\Gamma, \mathbb{Q}_{l}\right)$.

This example has a more classical interpretation. If we let $J$ be the Jacobian of $\bar{X}$, then the connected component of the identity in the special fiber of the Néron model of $J$ over $\mathcal{O}$ is an extension of an abelian variety by a torus; let $\chi$ 
be the character group of this torus. Then $\chi$ is naturally isomorphic to $H_{1}(\Gamma, \mathbb{Z})$. Moreover, one has a monodromy pairing $\chi \times \chi \rightarrow \mathbb{Z}$ (see [SGA] for details.) If one identifies $\chi$ with $H_{1}(\Gamma, \mathbb{Z})$, the resulting pairing on $H_{1}(\Gamma, \mathbb{Z})$ is precisely the length pairing.

To summarize:

Proposition 7.6. If $X$ is a schön open subset of a smooth proper curve $\bar{X}$ over $K$, then the "length pairing" on $\Gamma_{X}$ coincides with the monodromy pairing on the character group $\chi$ associated to the Jacobian of $\bar{X}$.

This has connections to Mikhalkin's construction of tropical Jacobians. Given a tropical curve $\Gamma$, which Mikhalkin interprets as a metric graph, the length pairing on $\Gamma$ induces a map $H_{1}(\Gamma) \rightarrow \operatorname{Hom}\left(H_{1}(\Gamma), \mathbb{Z}\right)$; Mikhalkin defines the tropical Jacobian of $\Gamma$ to be the torus $\operatorname{Hom}\left(H_{1}(\Gamma), \mathbb{R}\right) / H_{1}(\Gamma)$. This torus has a natural integral affine structure induced from that on $\operatorname{Hom}\left(H_{1}(\Gamma), \mathbb{R}\right)$. See $\mathbb{M Z}$ for details.

Mikhalkin's definition is purely combinatorial but has a nice interpretation in terms of the uniformization of abelian varieties: if $J$ is the Jacobian of $\bar{X}$ then there is a pairing $\chi \times \chi \rightarrow \bar{K}^{*}$ whose valuation is the monodromy pairing. This pairing gives an embedding of $\chi$ as a lattice in the torus $\operatorname{Hom}\left(\chi, \bar{K}^{*}\right)$; the quotient $\operatorname{Hom}\left(\chi, \bar{K}^{*}\right) / \chi$ is a rigid space isomorphic to $J$. If we "tropicalize" this space by taking valuations, we obtain the space $\operatorname{Hom}(\chi, \mathbb{R}) / \chi$, where $\chi$ embeds into $\operatorname{Hom}(\chi, \mathbb{R})$ by the monodromy pairing. In particular the "tropicalization" of $J$ is the tropical Jacobian of $\Gamma_{X}$.

The upshot is that- provided we are careful about what we mean by tropicalization"tropicalization" commutes with taking Jacobians.

The following result of [KMM] is another easy consequence of this point of view:

Proposition 7.7 ( $\mathrm{KMM}$, Theorem 6.4). Let $X$ be a schön open subset of an elliptic curve $\bar{X}$ over $K$ with potentially multiplicative reduction. Then $H_{1}\left(\Gamma_{X}, \mathbb{Z}\right)$ is isomorphic to $\mathbb{Z}$, and valuation of the $j$-invariant $j(\bar{X})$ is equal to $-a$, where a is the length of the unique cycle in $\Gamma_{X}$.

Proof. Replacing $\mathcal{O}$ with a suitable ramified extension we may assume that $\Gamma_{X}$ is integral. Then $\bar{X}$ has split multiplicative reduction. This base change scales both $\Gamma_{X}$ and the valuation of $j(\bar{X})$ by the degree $d$ of the extension. Now the tropical degeneration $\mathcal{X}$ of $X$ associated to $\Gamma_{X}$ gives a model of $\bar{X}$ whose special fiber contains a cycle of rational curves, of length equal to the lattice length $a$ of the unique cycle in $\Gamma_{X}$. The conductor-discriminant formula ([Si], Theorem 11.1) then shows that the valuation of $j(X)$ is equal to $-a$.

In fact, it is easy to see that any smooth curve $\bar{X}$ contains a schön open subset: take a semistable model of $\bar{X}$, embed it in $\mathbb{P}_{\mathcal{O}}^{n}$, let $\mathcal{T}$ be the complement of $n+1$ hyperplanes in general positionin $\mathbb{P}_{\mathcal{O}}^{n}$, and take $X=\mathcal{T} \cap \bar{X}$. Then the compactification $\bar{X}$ of $X$ in $\mathbb{P}_{\mathcal{O}}^{n}$ is tropical, and one verifies easily that the multiplication map is smooth. Thus the above result applies to all elliptic curves with potentially multiplicative reduction. Luxton and $\mathrm{Qu}$ LQ have shown that any variety over a field of characteristic 0 contains a schön open subset.

\section{Complete Intersections}

In the constant coefficient case, a (Zariski) general hyperplane section of a schön variety is schön. Unfortunately this is no longer true in the nonconstant coefficient 
case. For instance, let $X_{k}$ be a singular hypersurface in $T_{k}$. Then any hypersurface $X$ in $T_{K}$ that reduces modulo $\pi$ to $X_{k}$ has in $\operatorname{in}_{(0, \ldots, 0)} X=X_{k}$, and hence cannot be schön. The set of such $X$ is a rigid analytic open subset of the projective space of hypersurfaces of fixed degree.

As this example suggests, to study loci of schön varieties in a nonconstant coefficient setting, one needs to work with the rigid analytic topology rather than the Zariski topology. (For the basics of the theory of rigid analytic spaces we refer the reader to [EKL] or $[\mathrm{Sch}$; we use very little here.)

To make precise the connection to rigid geometry, we first observe:

Lemma 8.1. Let $\mathbb{P}$ be a toric scheme, proper over $\mathcal{O}$, and let $X$ be a subvariety of the open torus $T$ in $\mathbb{P} \times_{\mathcal{O}}$ Spec $K$. Suppose that for all polyhedra $P$ in the polyhedral complex $\Sigma$ corresponding to $P$, the closure $\mathcal{X}$ of $X$ in $\mathbb{P}$ intersects $\mathbb{P}_{P}$ transversely. Then $X$ is schön, and $\left(X, \mathbb{P}^{\prime}\right)$ is a normal crossings pair, where $\mathbb{P}^{\prime}$ is the open subset of $\mathbb{P}$ obtained by deleting all torus orbits that do not meet $\mathcal{X}$.

Conversely, if $X$ is schön and there exists a toric open subset $\mathbb{P}^{\prime}$ of $\mathbb{P}$ such that $\left(X, \mathbb{P}^{\prime}\right)$ is a normal crossings pair, then the closure of $X$ intersects $\mathbb{P}_{P}$ transversely for all polyhedra $P$ in $\Sigma$.

Proof. Consider the multiplication map

$$
m: \mathcal{T} \times \mathcal{X} \rightarrow \mathbb{P}^{\prime} .
$$

If $y$ is a point in $\mathbb{P}^{\prime}$ in the torus orbit corresponding to a polyhedron $P$ in the subcomplex $\Sigma^{\prime}$ of $\Sigma$ corresponding to $\mathbb{P}^{\prime}$, then the fiber over $y$ is isomorphic to the product $\mathcal{X} \cap \mathbb{P}_{P}^{\prime}$ with a torus. By assumption, this is smooth, so $m$ has smooth fibers. The argument of $[\mathrm{H}]$, Lemma 2.6 then shows that $m$ is smooth. It follows that $X$ is schön and $\left(X, \mathbb{P}^{\prime}\right)$ is a normal crossings pair. The converse is clear.

Note that the lemma implies that $\operatorname{Trop}(X)$ will be equal to the support of $\Sigma^{\prime}$ for all such $X$. One can therefore use this result to study the space of schön subvarieties of a toric variety over $K$ with a given tropicalization. We will not pursue this here, beyond a few straightforward observations.

Suppose $\mathbb{P}$ is projective. Fix an $X$ as in the lemma, and let $\operatorname{Hilb}(\mathbb{P})$ be the Hilbert scheme over $\mathcal{O}$ parameterizing subschemes of $\mathbb{P}$ with the same Hilbert polynomial as the closure of $X$. Complex points of $\operatorname{Hilb}(\mathbb{P})$ correspond to subschemes of the special fiber of $\mathbb{P}$; those that meet each $\mathbb{P}_{P}$ transversely form an open subset $U_{0}$ of $\operatorname{Hilb}(\mathbb{P}) \times_{\mathcal{O}} \operatorname{Spec} k$.

Now if $y$ is a point of $\operatorname{Hilb}(\mathbb{P})(\bar{K})$, then $y$ corresponds to a subscheme $X_{y}$ of the general fiber of $\mathbb{P}$ over a finite extension of $K$. Then $X_{y} \cap T$ will satisfy the hypotheses of Lemma 8.1 if, and only if, $y$ specializes to a point $y_{0}$ on the special fiber of $\operatorname{Hilb}(\mathbb{P})$ that lies in $U_{0}$. The set of points $y$ that specialize to $U_{0}$ forms a "neighborhood of $U_{0}$ " in the rigid analytic topology on Hilb $(\mathbb{P})$. More precisely, let $\operatorname{Hilb}(\mathbb{P})^{\text {rig }}$ denote the rigid analytic space associated to the general fiber of $\operatorname{Hilb}(\mathbb{P})$; then $\operatorname{Hilb}(\mathbb{P})^{\text {rig }}$ is equipped with a "reduction $\bmod \pi$ " map

$$
\operatorname{Hilb}(\mathbb{P})^{\text {rig }} \rightarrow \operatorname{Hilb}(\mathbb{P}) \times_{\mathcal{O}} \operatorname{Spec} k .
$$

The preimage of $U_{0}$ under this map is an admissible open subset $U^{\mathrm{rig}}$ of $\operatorname{Hilb}(\mathbb{P})^{\mathrm{rig}}$, and those $y \in \operatorname{Hilb}(\mathbb{P})(\bar{K})$ such that $X_{y} \cap T$ satisfies the hypotheses of Lemma 8.1 are precisely the $\bar{K}$-points of $U^{\text {rig. }}$.

If we restrict our attention to complete intersections, we can say more than this. In particular fix a projective toric scheme $\mathbb{P}$ over $\mathcal{O}$, and ample line bundles 
$L_{1}, \ldots, L_{s}$ on $\mathbb{P}$. The space $\mathcal{H}$ parameterizing tuples $\left(D_{1}, \ldots, D_{s}\right)$ such that for each $i, D_{i}$ is an effective divisor in the linear system corresponding to $L_{i}$, and all the $D_{i}$ 's intersect transversely, is an open subset of a product of projective spaces over $\mathcal{O}$.

By Bertini's theorem, the set of points in $\mathcal{H}(k)$ that correspond to divisors $\left(D_{1}, \ldots, D_{s}\right)$ in $\mathbb{P} \times_{\mathcal{O}}$ Spec $k$ such that $D_{1} \cap \cdots \cap D_{s}$ intersects each stratum $\mathbb{P}_{P}$ of $\mathbb{P}$ transversely is an open dense subset $U_{0}$ of the special fiber of $\mathcal{H}$. The preimage of $U_{0}$ under the reduction map

$$
\mathcal{H}^{\text {rig }} \rightarrow \mathcal{H} \times \times_{\mathcal{O}} \operatorname{Spec} k
$$

is a (necessarily nonempty) admissible open subset $U^{\text {rig }}$ of $\mathcal{H}^{\text {rig }}$; the points of $U^{\text {rig }}$ correspond precisely to those complete intersections $D_{1} \cap \cdots \cap D_{s}$ whose intersection with $T$ satisfies the conditions of Lemma 8.1.

Moreover, if $\left(D_{1}, \ldots, D_{s}\right)$ is a $K$-point of $U^{\text {rig }}$, and $X$ is the corresponding complete intersection $D_{1} \cap \cdots \cap D_{s} \cap T$ in $T$, then for each polyhedron $P$ in $\Sigma$, $\mathcal{X}_{P}=D_{1} \cap \cdots \cap D_{s} \cap \mathbb{P}_{P}$ is the intersection of ample divisors in the smooth toric variety $\mathbb{P}_{P}$, and is therefore either zero-dimensional or connected.

Lemma 4.5 and Theorem 6.1 now have immediate implications for the cohomology of $\operatorname{Trop}(X)$ :

Theorem 8.2. Let $\left(D_{1}, \ldots, D_{s}\right)$ be a $K$-point of $U^{\mathrm{rig}}$, and set

$$
X=D_{1} \cap \cdots \cap D_{s} \cap T \text {. }
$$

Then $H^{r}\left(\operatorname{Trop}(X), \mathbb{Q}_{l}\right)$ vanishes for $1 \leq r<\operatorname{dim} X$, and the natural map:

$$
H^{r}\left(\operatorname{Trop}(X), \mathbb{Q}_{l}\right) \rightarrow W_{0} H_{\text {ét }}^{r}\left(\bar{X}_{K^{\mathrm{sep}}}, \mathbb{Q}_{l}\right)
$$

is injective for $r=\operatorname{dim} X$.

Proof. The above discussion shows that $X$ is schön and $(X, \mathbb{P})$ is a normal crossings pair. We thus apply Theorem 6.1 and Lemma 4.5 to see that the map

$$
H^{r}\left(\operatorname{Trop}(X), \mathbb{Q}_{l}\right) \rightarrow W_{0} H_{\text {ét }}^{r}\left(\bar{X}_{K^{\text {sep }}}, \mathbb{Q}_{l}\right)
$$

is an isomorphism for $0 \leq r<\operatorname{dim} X$ and injective for $r=\operatorname{dim} X$. On the other hand, $\bar{X}$ is a complete intersection in the general fiber of the smooth toric variety $\mathbb{P} \times_{\mathcal{O}}$ Spec $K$. The result thus follows from Corollary 6.3

\section{REFERENCES}

[B] Berkovich, V. An analog of the Tate conjecture over local and finitely generated fields, Internat. Math. Res. Notices (2000), no. 13,665-680.

[BGS] J. Burgos Gil and M. Sombra. When do the recession cones of a polyhedral complex form a fan? preprint, arXiv:1008.2608

[dJ] de Jong, A. J. Smoothness, semistability, and alterations, Inst. Hautes Études Sci. Publ. Math. No. 83, (1996), 51-93.

[D] Deligne, P. La conjecture de Weil II, Inst. Hautes Études Sci. Publ. Math. no. 52 (1980), $137-252$.

[EKL] Einsiedler, M., Kapranov, M., and Lind, D. Non-archimedean amoebas and tropical varieties, J. reine und angewandte Mathematik 601 (2006) 139-157.

[F] Fulton, W. Introduction to Toric Varieties, Princeton University Press, 1993.

[GS] Gross, M., Siebert, B. Mirror Symmetry via Logarithmic Degeneration Data II, preprint arXiv:0709.2290

[H] Hacking, P. The homology of tropical varieties, Collect. Math. 59 (2008), no. 3, 263-273.

[HKT] Hacking, P., Keel, S., and Tevelev, J. Stable pair, tropical, and log canonical compact moduli of del Pezzo surfaces, Invent. Math. 178 (2009), no. 1, 173-227. 
[I] Ito, T. Weight-monodromy conjecture over equal characteristic local fields, Amer. J. Math. 127 (2005), no. 3, 647-658.

[KKMS] Kempf, G., Knudsen, F., Mumford, D., and Saint-Donat, B. Toroidal embeddings. I. Lecture Notes in Mathematics, Vol. 339. Springer-Verlag, Berlin-New York, 1973.

[KMM] Katz, E., Markwig, H., and Markwig, T. The tropical $j$-invariant, LMS J. Comput. Math. 12 (2009), 275-294.

[KS10] Katz, E. and Stapledon, A. The tropical motivic nearby fiber, preprint, arXiv:1007.0411.

[KS06] Kontsevich, M., and Soibelman, Y. Affine structures and non-Archimedean analytic spaces. The unity of mathematics, 321-385, Birkhäuser Boston, Boston, MA, 2006.

[LQ] Luxton, M. and Qu, Z. On Tropical Compactification. preprint, 0902.2009.

[MZ] Mikhalkin, G., and Zharkov, I. Tropical curves, their Jacobians, and theta functions. Curves and abelian varieties, 203-230, Contemp. Math., 465, Amer. Math. Soc., Providence, RI, 2008.

[NS] Nishinou, T., Siebert, B. Toric degenerations of toric varieties and tropical curves, Duke Math. J. 135 (2006), no. 1, 1-51.

[P] Payne, S. Analytification is the limit of all tropicalizations, Math. Res. Lett. 16 (2009), no. 3, 543-556.

[RZ] Rapoport, M., Zink, T. Über die lokale Zetafunktion von Shimuravarietäten, Monodromiefiltrations und verschwindende Zyklen in ungleicher Characteristik, Inv. Math. 68 (1980), 21-101.

[Si] Silverman, J. Advanced topics in the arithmetic of elliptic curves. Graduate Texts in Mathematics, 241, Springer-Verlag, New York, 1994.

[S] Smirnov, A. L. Torus schemes over a discrete valuation ring, Algebra i Analiz 8 (1996), no. 4, 161-172; translation in St. Petersburg Math. J. 8 (1997), no. 4, 651-659.

[Sch] Schneider, P. Basic notions of rigid analytic geometry, in Galois Representations in Arithmetic Algebraic Geometry (Durham, 1996), London Math. Soc. Lecture Note Ser. 254, Cambridge University Press (1998), 369-379.

[SGA] Grothendieck, A. Modèles de Néron et monodromie SGA 7 I, Exposé IX. 313-523, Lecture Notes in Math. 288, Springer-Verlag, New York, 1972.

[Sp1] Speyer, D. Tropical Geometry, PhD thesis, UC Berkeley, 2005.

[Sp2] Speyer, D. Uniformizing tropical curves I: genus zero and one, preprint arXiv:0711.2677.

[T] Tevelev, J. Compactifications of subvarieties of tori, Amer. J. Math. 129 (2007), no. 4, 10871104.

Department of Mathematics, University of Texas at Austin, 1 University Station C1200, Austin, TX 78712-0257 